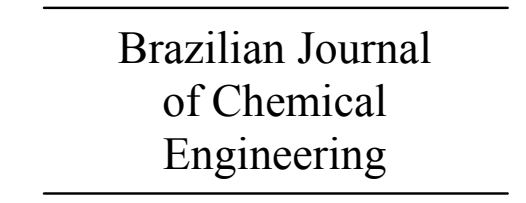

ISSN 0104-6632

Printed in Brazil

www.abeq.org.br/bjche

Vol. 30, No. 03, pp. 413 - 435, July - September, 2013

\title{
INVESTIGATION OF HEAT TRANSFER PROCESSES INVOLVED LIQUID IMPINGEMENT JETS: A REVIEW
}

\author{
M. Molana and S. Banooni* \\ Department of Mechanical Engineering, Engineering Faculty, Phone: 0098 (662) 5304043, \\ Fax: 0098 (611) 336-9684, Shahid Chamran University, Ahvaz, Iran. \\ E-mail: maysammolana@yahoo.com \\ Department of Mechanical Engineering, Engineering Faculty, Phone: +98 (912) 3885209, \\ Fax: 0098 (611) 336-9684, Shahid Chamran University, Ahvaz, Iran. \\ E-mail: banooni@scu.ac.ir
}

(Submitted: April 28, 2012 ; Revised: July 18, 2012 ; Accepted: July 27, 2012)

\begin{abstract}
This review reports research on liquid impingement jets and the abilities, limitations and features of this method of heat transfer. Some available and important correlations for Nusselt number are collected here. Also we demonstrate the capability of nanofluids to be applied in heat transfer processes involved liquid impingement jets.

Keywords: Liquid impingement jet; Nanofluid; Heat transfer; Nusselt number.
\end{abstract}

\section{INTRODUCTION}

Impinging jets can be classified as either freesurface or submerged. Submerged jets exude into a space containing the same liquid at rest and can be configured as confined or unconfined, depending on the jet-to-target distance. In a submerged configuration, the interaction of the issuing jet and the stagnant fluid leads to entrainment in the shear zone and the development of a potential core near the jet centerline. The potential core is the region in which the velocity of the jet remains largely unaffected by the spreading of the jet due to entrainment (Webb and Ma, 1995). In the confined case, the fluid heated by the target plate can, in some instances, get recirculated and be entrained back into the impinging jet. This causes the formation of a re-circulation zone in the outflow region (Fitzgerald and Garimella, 1998). In an unconfined configuration, such as the case when jets are issuing from long tubes, the jet interacts with the ambient and otherwise quiescent surroundings and has associated with it higher heat transfer coefficients because the heated fluid is not entrained back into the jet, as is sometimes the case for confined jets (Lupton et al., 2008). The point at which jets differentiate between fully confined and fully unconfined will likely depend on many factors, including but not limited to $\mathrm{Re}, \mathrm{H} / \mathrm{d}$ and surface topology. Although this has not been fully characterized, some progress has been made (Lupton et al., 2008).

In a free-surface jet configuration, the fluid is discharged from the nozzle into an ambient gas, typically air, before impinging upon the target surface. Therefore, entrainment of the surrounding fluid can be considered to be negligible so that a potential core is not relevant in this case. Due to the intensive trend of component miniaturization, the available space for the cooling device will likely be very restricted. Different types of jets are showed in Figure 1.

*To whom correspondence should be addressed 

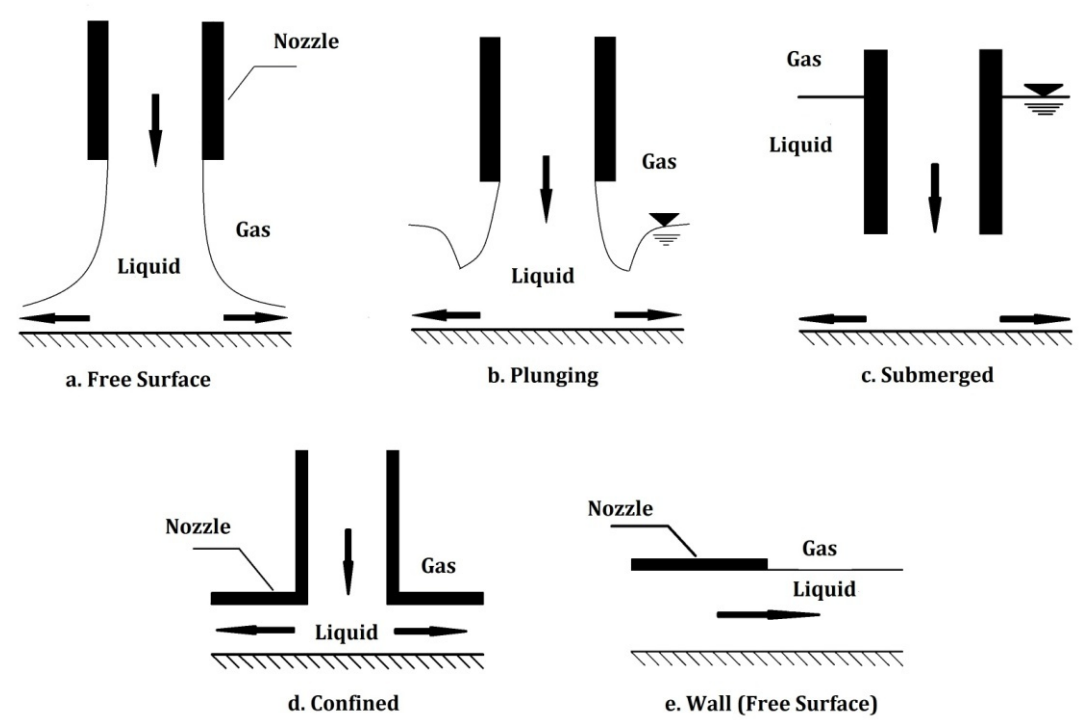

Figure 1: Different types of impingement jets.

The magnitude and distribution of the heat transfer coefficient has been found to be dependent on a number of parameters, including Prandtl number (Pr), Reynolds number (Re), jet-to-target spacing $(\mathrm{H} / \mathrm{d})$, jet diameter (d) and the physical geometry of the jets and heater surface (Martin, 1977), (Jambunathan et al., 1992). Compared to single jet impingement, the heat transfer and flow characteristics of jet arrays have received much less attention in the open literature, although some works exist (Goldstein and Timmers, 1982).

The primary advantage of arrays of multiple jets is the creation of multiple stagnation zones with sometimes exceptionally high local heat transfer coefficients. Depending on aspects such as jet to jet spacing and cross flow conditions, high local surface heat transfer coefficients can be maintained over the entire surface. This can result in very high surface average heat transfer coefficients and more uniform surface temperature distributions, which is a particularly desirable property of an electronics cooling package. The use of impinging jets as an interesting method of heat removal is one area receiving some consideration. Impingement jets have already found extensive use in industrial applications for purposes such as the thermal treatment of metals and the cooling of turbine blades (Schutt, 2007; Bunker, 2007) solar collectors, oil jets in oil cooled internal combustion engines (Kiryo, 1986), water jets in the hot rolling process of steel (Kohing, 1985), medical cryosurgery of cancers (Aihara and Kim, 1993), X-ray devices and military transient cooling in rocket nozzles (Kandlikar, 1998) and laser weapons (Mudawar and Wadsworth, 1991).
Banooni et al. (2008) studied heat transfer effects of an impingement jet on baking of flat bread. They concentrated on some important parameters such as jet velocity variation, jet temperature, jet to target distance and quality properties of bread as target. They demonstrated clearly the desirability of using impingement jets in baking, because of heat transfer uniformity and better quality properties of bread. Therefore, this heat transfer approach is a promising method in food processing.

The popularity of impingement jet systems derives from the fact that they possess one of the highest known single phase heat transfer coefficients. A few studies reviewed heat transfer of impingement jets and their applications (Polat et al., 1989), (Jones and Launder, 1972). In this study, we concentrate on works related to liquid impingement jets and their applications for cooling and heating in recent years. Also we focus on the types of the jets and their effects on the heat transfer coefficient.

\section{HYDRODYNAMICS OF LIQUID IMPINGEMENT JETS}

Liquid impingement jet characteristics are similar to gas impingement jets with a few differences. When a liquid jet is impinged on a target, three distinct regions are identified. The first zone is the free jet region, which develops instantly at the nozzle exit and remains throughout the impingement process. When an axial free surface jet impinges on a target, the fluid forms a boundary layer, which grows along the target dimensions (for example radius, for a 
circular disk as target). The second zone is the impingement region, where the interaction between the jet and the heated target produces a strong deceleration of the flow. After this zone, the liquid wets and flows in a direction parallel to the target.

The difference between liquid and gas impingement jets is that, when a turbulent liquid jet impinges on a flat target, a spray of droplet breaks off from the liquid layer formed on the target. This splattering of droplets lowers the efficiency of the impingement jet heat transfer cooling process due to the loss of liquid. The laminar jets do not splatter. When the jet turbulence is sufficiently large, these waves sharpen and break into droplets. All observations indicate that the amplitude of the disturbance on the jet governs splattering (Varella and Leinhard, 1991). It should be noted that the amount of splattering at a given nozzle to target spacing depends principally on the jet Weber number. Also, the surface tension of the liquid plays an important role in splattering (Bhunia, 1993). A liquid impingement jet is shown in Figure 2.

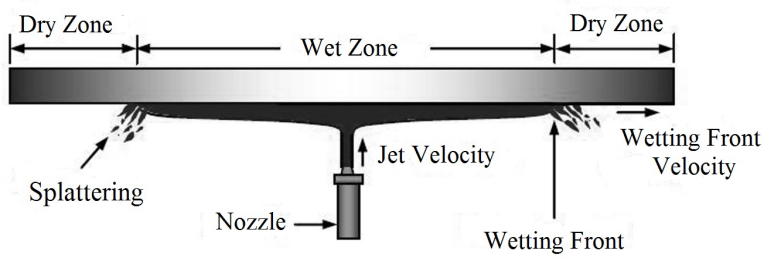

Figure 2: Liquid impingement jet

When a liquid is first impinged on the hot surface, it remains stagnant in a small impingement region for a certain period of time before covering the entire surface. This time period varies from a fraction of a second to a few minutes, which depends on the experimental conditions. This wetting delay period is described as the residence time, $t^{*}$, in the present study. The local wall temperature at the stagnation radius at the residence time is represented by $\mathrm{T}_{\mathrm{w}}^{*}$ in this study. Figure 3 describes the above mentioned definition of quenching phenomena by using a cooling curve. Just after the residence time, the wetting front starts moving and consequently the surface temperature drops at a faster rate. Before the residence time, the surface temperature drops slowly and almost at a constant rate though there is a sudden drop of temperature at the very beginning of the jet impingement.

Some studies (Liu et al., 1991; Leinhard et al., 1972) also performed on free surface jet impingement cooling, have given valuable background information on single phase convection heat transfer.
Wetting delay is an important phenomenon in jet impingement quenching. A number of comprehensive reviews on jet impingement are available in the literature (Cooper et al., 1993; Amano and Brandt, 1984).

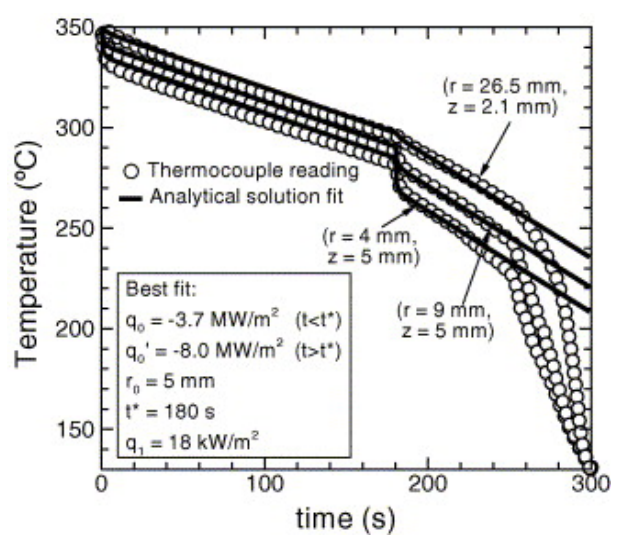

Figure 3: Cooling curves during quench for copper block $\mathrm{T}_{0}=350{ }^{\circ} \mathrm{C}, \mathrm{T}_{\text {liq }}=51^{\circ} \mathrm{C}$, uj $=3 \mathrm{~m} / \mathrm{s}$ (reprinted from Woodfield et al. (2005), with permission from Elsevier).

\section{SINGLE LIQUID IMPINGEMENT JETS}

This type of impingement jets have been investigated extensively numerically, experimentally and theoretically by many researchers and scientists for different applications. Watson (1964) solved the hydrodynamic problem of the impact of a liquid jet over a horizontal plane analytically.

In the experiment by Carper and Deffenbaugh (1978) a nozzle diameter of $0.2 \mathrm{~cm}$ was used for Reynolds number ranging from 200 to 400 , a nozzle diameter $0.4 \mathrm{~cm}$ was used for Re of 400 to 1000 and for $\mathrm{Re}$ of higher than 1000 a nozzle diameter of $0.8 \mathrm{~cm}$ was used. They compared their results with predictions from using Watson's solution. It must be mentioned that they concluded Nusselt number did not depend on the nozzle diameter. The Watson's analytical solution predicts Nusselt number that are in good agreement with experimental results for a nozzle diameter of $0.4 \mathrm{~cm}$ in the range of $\mathrm{Re}=400$ to 1000 . The results from using a nozzle diameter of $0.2 \mathrm{~cm}$ are lower and for a nozzle diameter of $0.8 \mathrm{~cm}$ the Nusselt numbers are higher than the Watson's solution.

A theoretical analysis was conducted to characterize heat transfer from horizontal surfaces to normally impinging slot jets under given heat flux conditions by Chen et al. (2005). The thermal and hydraulic boundary layers of laminar flow were 
divided into four regions of flow along heat transfer surfaces, including a stagnation zone and three wall jet zones, from which general expressions for the heat transfer coefficients were obtained. Furthermore, those results were compared with experimental and analytical data available in the published literature. Good agreement was observed from the comparisons.

Patel and Roy (2001) researched jet impingement heat transfer for varying fluid flow characteristics and concluded that:

- Heat transfer is maximum through the shear layer formed near the jet attachment stagnation region.

- For all Reynolds numbers, the maximum Nusselt number for the closed boundary condition is higher than for the open boundary condition. This is primarily because more energy is lost in the open boundary condition than in the closed adiabatic boundary conditions.

- Turbulence (velocity gradient) and wall shear force are higher in the shear layer region and highest at the stagnation point.

- The average Nusselt number grows as the Reynolds number increases.

The objective of Lallave et al.'s (2007) study was to characterize the conjugate heat transfer for a confined liquid jet impinging on a rotating and uniformly heated solid disk of finite thickness and radius. The model covered the entire fluid region (impinging jet and flow spreading out over the rotating surface) and the solid disk as a conjugate problem. It was found that plate materials with higher thermal conductivity maintained a more uniform temperature distribution at the solid-fluid interface. A higher Reynolds number increases the local heat transfer coefficient reduced the wall to fluid temperature difference over the entire interface. The rotational rate also increases the local heat transfer coefficient under most conditions. The simulation results compared reasonably well with previous experimental studies. They concluded that the solid-fluid dimensionless interface temperature, the local Nusselt number and the local heat transfer coefficient show a strong dependence on the following parameters: Reynolds number, rotational rate, impingement height, disk thickness, solid material properties, and fluid properties. Increasing the Reynolds number increases the local heat transfer coefficient distribution values over the entire solidfluid interface. Simultaneously, this effect reduces the solid-fluid temperature and improves the cooling effectiveness of the process.

Miller et al. (1995) performed a numerical study on non-circular jets. They simulated the performance of different geometries such as elliptic, rectangular and triangular jets with different aspect ratios.

Direct Numerical Simulation (DNS) of an unsteady impinging jet were performed by Chung et al. (2002) to study momentum and heat transfer characteristics. The unsteady compressible NavierStokes equations were solved using a high-order finite difference method with non-reflecting boundary conditions. It was found that the impingement heat transfer was very unsteady and the unsteadiness was caused by the primary vortices emanating from the jet nozzle. These primary vortices dominate the impinging jet flow as they approach the wall. Detailed analysis of the instantaneous flow and temperature fields was performed, showing that the location of primary vortices significantly affects the stagnation Nusselt number.

Thermochromic liquid crystals have been extensively applied to natural convection flows for the visualization of temperature and induced velocity fields. In a study by Ashforth-Frost and Rudel (2002), a single submerged impinging water jet was artificially seeded with microencapsulated liquid crystals. A nozzle to plate spacing of three nozzle diameters and a turbulent Reynolds number of 35500 was considered. Using liquid crystals as particle tracers had been demonstrated to be a useful tool for the simultaneous quantitative visualization of flow and temperature in a forced convection flow. Despite the hysteresis effects observed in the calibration of thermo-chromic liquid crystals, they have been demonstrated as a useful tool for gaining simultaneous insight into the hydrodynamic and thermal flow features of a turbulent impinging jet.

Teamah and Farahat (2003) studied heat transfer from impinging of a single free liquid jet and observed that, from the results obtained either numerically or experimentally, both local and average Nusselt numbers were higher in the shooting flow region than in the streaming flow region. This is because the fluid film thickness is very small in that region compared with the outer region. This means that the mean velocity of the fluid in that region is higher. It was also found from the comparison of the numerical and experimental results for single jet that there is a good agreement between them. Both the local and average Nusselt numbers increase with increasing Reynolds number.

Stevens and Webb (1991) performed experiments to study the heat transfer between a round jet impinging on a flat plate with a constant heat flux. Effects of the jet Reynolds number, nozzle to plate spacing and jet diameter on heat transfer were investigated. Increasing the nozzle spacing generally 
decreased the Nusselt number slightly. Correlations for the local and average Nusselt numbers revealed an approximate Nusselt number dependence on the Reynolds number raised to the third power.

Poh et al. (2005) investigated the effect of flow pulsations on the time-averaged Nusselt number for axis-symmetric semi-confined laminar impinging water jets. In that study, it was found that the onset of separation in the wall jet region of the pulsating impinging jet is associated with the point of constant Nusselt number during the oscillation cycle. This is attributed to the existence of vortices above that location, which tend to suppress the flow acceleration and deceleration phenomena during oscillation.

Conjugate heat transfer from a uniformly heated spinning solid disk of finite thickness and radius during a semi-confined liquid jet impingement from a rotating nozzle was studied by Rahman et al. (2008), who concluded that,

- A great disk thickness provides a more uniform interface temperature and heat transfer coefficient.

- A reduction in the plate to disk confinement ratio increases the local Nusselt number at all locations.

- A higher Prandtl number fluid leads to a thinner thermal boundary layer and provides a more effective heat removal rate at the solid - fluid interface.

- Zuckerman and Lior (2006) performed a comprehensive study on jet impingement heat transfer. They presented an interesting viewpoint in this area, including some disadvantages of impingement cooling devices, as follow:

- For moving targets with very uneven surfaces, the jet nozzles may have to be located too far from the surface. For jets starting at a large height above the target, the decay in kinetic energy of the jet as it travels to the surface may reduce average Nu by $20 \%$ or more.

- The hardware changes necessary for implementing an impinging jet device may degrade structural strength (one reason why impinging jet cooling is more easily applied to turbine stator blades than to rotor blades).

- In static applications where very uniform surface heat or mass transfer is required, the resulting high density of the jet array and corresponding small jet height may be impractical to construct and implement, and at small spacings jet-to-jet interaction may degrade efficiency.

\section{MULTIPLE LIQUID IMPINGEMENT JETS}

Robinson and Schnitzler (2007) conducted experiments investigating the impingement of water jet arrays under both free-surface and submerged conditions. In this work, a large population of jets was used, which induces cross flow which is known to influence the heat transfer (Obot and Trabold, 1987). Jet diameters of $1 \mathrm{~mm}$ were used, with a jetto-jet spacing range of $3<\mathrm{s} / \mathrm{D}<7$. Heater surfaceaveraged heat transfer and pressure drop data were recorded for a dimensionless jet-to-target distance range of $2<\mathrm{H} / \mathrm{D}<30$ and a Reynolds number range of $650<\operatorname{Re}_{\mathrm{D}}<6500$. For the submerged jets, it was found that heat transfer was insensitive to jetto-target distance changes in the range of $2<\mathrm{H} / \mathrm{D}<3$. A uniform decrease in heat transfer was observed with increasing jet-to-target distance in the range of $5<\mathrm{H} / \mathrm{D}<20$. It was reported that, for a constant Reynolds number, increasing the jet-to-jet spacing resulted in a detrimental effect on heat transfer. A stronger dependence on jet-to-jet spacing was encountered for smaller jet-to-target spacing. Free-surface jets were observed to behave as submerged jets within the range of $5<\mathrm{H} / \mathrm{D}<20$. Beyond this value, entirely free jet flow occurs, with the heat transfer coefficient showing marginal improvement with increasing jet-to-target distance. At a jet-to-target spacing below $\mathrm{H} / \mathrm{D}=3$ the confined-submerged jets provided higher heat transfer coefficients for lower pumping power cost compared with free jets. It was also noted that, under confined-submerged conditions, decreasing the interjet distance, and therefore increasing the number of jets, decreased the pumping power requirement for a given heat transfer coefficient.

Royne and Dey (2006) investigated the effect of nozzle geometry on the heat transfer and pressure drop of confined-submerged jet arrays. Four different geometries were investigated with sharp-edged nozzles. These results were attributed to their respective favorable and unfavorable discharge coefficients. With regards to heat transfer for a given pumping power, it was reported that the sharp-edged and contoured nozzles both experienced enhanced performances in comparison to the conventional straight nozzle arrays. The sharp-edged nozzle array decreased the flow rate required for a given heat transfer, while the countersunk nozzle required an increased flow rate at a reduced pressure drop.

Jiji and Dagan (1987) presented a useful correlation for the heater surface-average Nusselt number under impinging free-surface liquid jets. That empirical correlation incorporated a jet configuration factor that took into account the number and size of jets. In a well-drained array, cross flow between jets is not important, with each jet establishing its own 
flow cell independent of the other jets. Hence, the heat transfer over the area of the flow cell is the same as for single jet impingement and the only effect of more jets is to decrease the amount of nonstagnation cooling. This result was confirmed by the work of Pan and Webb (1995).

Liquid jet and spray impingement cooling were studied experimentally and compared in the nonboiling regime by Oliphant et al. (1998). For the commercial nozzle and experimental conditions used in the present study, spray cooling demonstrated a strong dependence on mass flux and it was proposed that droplet velocity also affected the heat transfer. The results show that jet velocity plays a significant role in impingement cooling. For a given liquid mass flux and jet array configuration (e.g., the seven-jet configuration), an increase in velocity increased the heat transfer rate. However, comparing the sevenand four-jet configurations at the same jet diameter and liquid mass flux, the heat transfer coefficient was larger for the four-jet configuration. The decrease in the number of jets would be expected to decrease the average heat transfer, since less of the surface is exposed to the high transport region in a stagnation zone.

The decrease in the number of jets at a fixed mass flux, however, is offset by an increase in the jet velocity due to the reduction in total orifice area. The net effect is an increased average heat transfer. This indicates that there is a tradeoff between more jets and a greater stagnation region area (with its associated high transport) and higher velocities for better stagnation point cooling. One possibility is to reduce the diameter of the jets as their number is increased so that the velocity remains unchanged. This would provide high stagnation point heat transfer over the entire surface. The optimization of liquid jet array variables is considered in detail by Maddox and Bar-Cohen (1994).

The pitch $\mathrm{p}_{\mathrm{jet}}$, or center-to-center positioning of jets in an array, determines the degree of jet interaction. For jets spaced at a pitch-to-diameter ratio of $\mathrm{p}_{\mathrm{jet}} / \mathrm{D}<4$, the jets show significant interaction. San and Lai (2001) showed that, for $H / D=2$, the interference persisted up to spacings of $p_{\text {jet }} / D=8$ or 10 and the maximal $\mathrm{Nu}$ occurred at $\mathrm{p}_{\mathrm{jet}} / \mathrm{D}=8$.

A cooling device based on jet impingement was proposed for cooling of densely packed photovoltaic cells under a high concentration. The device consists of an array of jets where the cooling fluid is drained around the sides in the direction normal to the surface. It is shown by Royne and Dey (2007) that the inherently non-uniform heat transfer distribution of jet arrays had little effect on the expected electrical performance of the PV array. They compared their results to Martin's model and found good agreement. They also proposed a model which predicts the pumping power required for an average heat transfer coefficient for different device configurations. The model predicts that a higher number of nozzles per unit area will perform better than a lower number of nozzles. It also predicts an optimal nozzle diameter for a given area and number of nozzles.

In another interesting study done by Wang et al. (2004), micromachined liquid jets were employed for cooling of VSLI chips. This study presents the design and fabrication of single-jets and multijet arrays with circular orifice diameters ranging from 40 to $76 \mathrm{~m}$, as well as integrated heater and temperature sensor test devices. They used three jet arrays to investigate heat transfer performance. A removal of $90 \mathrm{~W}$ was demonstrated using a four-jet array at a flow rate of $8 \mathrm{ml} / \mathrm{min}$ with a $100{ }^{\circ} \mathrm{C}$ temperature rise with jet diameters of $76 \mu \mathrm{m}$. Boiling heat transfer coefficients of approximately $3 \mathrm{~W} / \mathrm{cm}^{2}$ $\mathrm{C}$ and a wall superheat of $30^{\circ} \mathrm{C}$ in the experiments suggested the possibility of pool boiling. However, reducing the wall superheat and achieving higher heat removal rates requires visualization of the flow regimes and optimization of the flow rates to reduce flooding of the reservoir. Furthermore, optimization of the geometry is needed to minimize the large pressure drops in the jet formation process and alternate geometries, such as square, elliptical, and slot orifices, and optimal orifice lengths and orifice to impingement plane spacing should be explored to increase heat removal rates.

Zuckerman and Lior (2007) simulated heat transfer of an array of slot jets using the $v^{2} f$ RANS model. They observed that the highest mean Nusselt number per unit of power or flow invested would occur will a lower number of jets ( $\mathrm{n}=2$ or 4$)$. Cooling arrangements with six and eight nozzles had similar levels of non-uniformity of the Nusselt number. They provided a predicting correlation for the Nusselt number that included the jet-to-cylinder diameter ratio, Reynolds number, number of jets, and the Prandtl number. They also concluded that increasing $\mathrm{n}$ at a given Reynolds number tended to increase the mean Nusselt number. Some available correlations for Nusselt number related liquid impingement jets have been collected in Table 1 . 
Table 1: Correlations for Nusselt number in liquid impingment jet systems

\begin{tabular}{|c|c|c|c|c|c|c|}
\hline Ref. No. & Type of jet & $\begin{array}{l}\text { Nozzle } \\
\text { geometry }\end{array}$ & Nusselt Correlation & Reynolds range & Type of Study & Notes \\
\hline $\begin{array}{l}\text { Chen et al. } \\
(2005)\end{array}$ & Free Surface & Slot & $\mathrm{Nu}=\left[\frac{2 \varepsilon}{\operatorname{PrRe}}+\frac{272 \mathrm{H}}{525 \mathrm{~B}}\right]^{-1}, \mathrm{H}=3.858 \frac{\mathrm{x}}{\operatorname{Re}}+\left(0.8-\frac{0.6277}{\mathrm{Re}^{0.305}}\right)$ & $\operatorname{Re}<100000$ & Theoretical & - \\
\hline $\begin{array}{l}\text { Teameh and } \\
\text { Farahat } \\
(2003)\end{array}$ & Free Surface & Circular & $\overline{\mathrm{Nu}}=10^{-2.25223}(\mathrm{r} / \mathrm{d})^{-1.15607} \mathrm{Re}^{1.1528288}$ & $25000<\operatorname{Re}<40000$ & $\begin{array}{l}\text { Experimental } \\
\text { and } \\
\text { Numerical }\end{array}$ & $3.6<\mathrm{r} / \mathrm{d}<54$ \\
\hline $\begin{array}{l}\text { Teameh and } \\
\text { Farahat } \\
(2003)\end{array}$ & Free Surface & Circular & $\overline{\mathrm{Nu}}=10^{0.755395}(\mathrm{r} / \mathrm{d})^{-1.50812} \mathrm{Re}^{0.56188}$ & $5000<\operatorname{Re}<20000$ & $\begin{array}{l}\text { Experimental } \\
\text { and } \\
\text { Numerical }\end{array}$ & $3.6<\mathrm{r} / \mathrm{d}<54$ \\
\hline $\begin{array}{l}\text { Stevens et al. } \\
(1991)\end{array}$ & Free Surface & Circular & $\mathrm{Nu}=0.93 \operatorname{Re}_{\mathrm{d}}^{1 / 2} \operatorname{Pr}^{0.4}$ & Not mentioned & Numerical & Turbulent flow \\
\hline $\begin{array}{l}\text { Rahman et al. } \\
\text { (2008) }\end{array}$ & Semi-confined & Circular & $\begin{array}{c}\overline{\mathrm{Nu}}=1.94282 \beta^{0.111} \mathrm{Re}^{0.75} \mathrm{Ek}_{1}^{-0.0465} \\
\mathrm{Ek}_{2}^{-0.047} . . \varepsilon^{-0.69}\left(\mathrm{r}_{\mathrm{p}} / \mathrm{r}_{\mathrm{d}}\right)^{-0.05}\end{array}$ & $220<\operatorname{Re}<900$ & Numerical & $\begin{array}{l}\text { Uniformly heated Spinning } \\
\text { solid disk; Rotating nozzle }\end{array}$ \\
\hline $\begin{array}{l}\text { Robinson and } \\
\text { Schnitzler } \\
(2007)\end{array}$ & $\begin{array}{l}\text { Free and } \\
\text { Submerged }\end{array}$ & Circular & $\begin{array}{l}\frac{N u}{\operatorname{Pr}^{0.4}}=7.8 \operatorname{Re}_{d_{n}}^{0.49} \exp \left(-0.025 \frac{\mathrm{S}}{d_{n}}\right) \\
\frac{N u}{\operatorname{Pr}^{0.4}}=23.39 \operatorname{Re}_{d_{n}}^{0.46}\left(\frac{\mathrm{S}}{d_{n}}\right)^{-0.442}\left(\frac{H}{d_{n}}\right)^{-0.00716}\end{array}$ & $100<\operatorname{Re}<10000$ & Experimental & $\begin{array}{l}\text { Miniature jet array } \\
2 \leq \mathrm{H} / \mathrm{dn} \leq 3\end{array}$ \\
\hline $\begin{array}{l}\text { Jiji and Dagan } \\
\text { (1987) }\end{array}$ & Free Surface & Circular & $\overline{\mathrm{Nu}}=3.84 \operatorname{Re}^{1 / 2} \operatorname{Pr}^{1 / 3}\left[0.008 \frac{\mathrm{Ln}}{\mathrm{d}}+1\right]$ & Not mentioned & Experimental & Multijet \\
\hline $\begin{array}{l}\text { Pan and Webb } \\
\text { (1993) }\end{array}$ & Free Surface & Circular & $\overline{\mathrm{Nu}}=0.225 \operatorname{Re}^{2 / 3} \operatorname{Pr}^{1 / 3} \mathrm{e}^{-0.095(\mathrm{~s} / \mathrm{d})}$ & Not mentioned & Numerical & Arrays of free-surface jets \\
\hline $\begin{array}{l}\text { Zuckerman } \\
\text { and Lior } \\
(2007)\end{array}$ & Free Surface & Slot & $\mathrm{Nu}_{\mathrm{av}}=0.12\left(\frac{\mathrm{d}}{\mathrm{D}}\right)^{-0.16}(\mathrm{n})^{0.18}(\mathrm{Re})^{0.66}(\mathrm{Pr})^{0.5}$ & $5000<\operatorname{Re}<80000$ & Numerical & $\begin{array}{l}2 \leq \mathrm{n} \leq 8 \\
0.7 \leq \operatorname{Pr} \leq 5.85 \\
2 \leq \mathrm{H} / 2 \mathrm{~B} \leq 5\end{array}$ \\
\hline $\begin{array}{l}\text { Patel and Roy } \\
\text { (2001) }\end{array}$ & Free surface & Rectangular & $\begin{array}{l}\mathrm{Nu}=-\left(0.0436 \mathrm{Re}^{2}+0.021 \mathrm{Re}+0.0081\right) \theta^{2} \\
\left(5.5 \operatorname{Re}^{2}+2.77 \mathrm{Re}+2.45\right) \theta \\
(103.2 \mathrm{Re}+140.4-130.8)\end{array}$ & $5000 \leq \operatorname{Re} \leq 20000$ & Numerical & $\begin{array}{l}\text { Jet angle } 30^{\circ} \leq \theta \leq 90^{\circ} \\
\text { a Pair of Rectangular Jets }\end{array}$ \\
\hline $\begin{array}{l}\text { Lallave et al. } \\
\text { (2007) }\end{array}$ & Confined & Circular & $\overline{\mathrm{Nu}}=1.97619 \beta^{0.0909} \mathrm{Re}^{0.75} \mathrm{Ek}^{-0.1111} \varepsilon^{-0.9}$ & $500<\operatorname{Re}<1500$ & Numerical & Target: rotating disk \\
\hline $\begin{array}{l}\text { Fang and } \\
\text { Kasagi (2007) }\end{array}$ & Free Surface & Circular & $\mathrm{Nu}_{\mathrm{o}}=0.409 \mathrm{Re}^{0.559} \operatorname{Pr}^{1 / 3}$ & $849<\operatorname{Re}<2124$ & Experimental & Micro-scale jet \\
\hline $\begin{array}{l}\text { Liu and } \\
\text { Gabour } \\
(1993)\end{array}$ & Confined & Circular & $\mathrm{Nu}_{\mathrm{o}}=0.797 \operatorname{Re}^{1 / 2} \operatorname{Pr}{ }^{1 / 3}$ & Not mentioned & Numerical & Laminar jets \\
\hline $\begin{array}{l}\text { Wolf et al. } \\
(1995)\end{array}$ & Free Surface & Circular & $\mathrm{Nu}_{\mathrm{o}}=0.202 \mathrm{Re}^{0.620} \operatorname{Pr}^{0.4}$ & Not mentioned & Experimental & $\begin{array}{l}\text { Turbulence dissipation in a } \\
\text { free surface jet of water }\end{array}$ \\
\hline $\begin{array}{l}\text { Vader et al. } \\
\text { (1991) }\end{array}$ & Confined & Planar & $\mathrm{Nu}_{\mathrm{o}}=0.28 \operatorname{Re}^{0.58} \operatorname{Pr}^{0.4}$ & $20000<\operatorname{Re}<90000$ & Numerical & $2.7<\operatorname{Pr}<4.5$ \\
\hline $\begin{array}{l}\text { Womac et al. } \\
\text { (1993) }\end{array}$ & Submerged & Circular & $\begin{array}{l}\frac{\mathrm{Nu}}{\operatorname{Pr}^{0.4}}=\mathrm{C}_{1} \operatorname{Re}_{\mathrm{d}}^{\mathrm{m}} \frac{1}{\mathrm{~d}} \mathrm{~A}_{\mathrm{r}}+\mathrm{C}_{2} \operatorname{Re}_{\mathrm{L}}^{\mathrm{n}} \frac{1}{\mathrm{~L}}\left(1-\mathrm{A}_{\mathrm{r}}\right) \\
\mathrm{L}=\frac{(0.5 \sqrt{2} \mathrm{l}-1.9 \mathrm{~d})+(0.51-1.9 \mathrm{~d})}{2} \mathrm{~A}_{\mathrm{r}}=\pi(1.9 \mathrm{~d})^{2} / 1^{2}\end{array}$ & $\operatorname{Re}<50000$ & Numerical & $1.65 \leq \mathrm{d} \leq 6.55 \mathrm{~mm}$ \\
\hline $\begin{array}{l}\text { Womac et al. } \\
\text { (1993) }\end{array}$ & Free Surface & Circular & $\begin{array}{l}\frac{\mathrm{Nu}}{\operatorname{Pr}^{0.4}}=\mathrm{C}_{1} \operatorname{Re}_{\mathrm{d}_{\mathrm{i}}}^{\mathrm{m}} \frac{1}{\mathrm{~d}_{\mathrm{i}}} \mathrm{A}_{\mathrm{r}}+\mathrm{C}_{2} \operatorname{Re}_{\mathrm{L}}^{\mathrm{n}} \frac{1}{\mathrm{~L}}\left(1-\mathrm{A}_{\mathrm{r}}\right) \\
\mathrm{L}=\frac{\left(0.5 \sqrt{2} 1-\mathrm{d}_{\mathrm{i}}\right)+\left(0.51-\mathrm{d}_{\mathrm{i}}\right)}{2} \mathrm{~A}_{\mathrm{r}}=\pi \mathrm{d}_{\mathrm{i}}^{2} / \mathrm{l}^{2}\end{array}$ & $\mathrm{Re}<50000$ & Experimental & $\begin{array}{l}1.65 \leq \mathrm{d} \leq 6.55 \mathrm{~mm} \\
3.5 \leq \mathrm{S}_{\mathrm{NP}} / \mathrm{d} \leq 10 \\
\mathrm{l}=12.7 \mathrm{~mm}, \mathrm{~m}=0.5 \\
\mathrm{n}=0.532, \mathrm{C}_{1}=0.516 \\
\mathrm{C}_{2}=0.491\end{array}$ \\
\hline $\begin{array}{l}\text { Silverman and } \\
\text { Nagler (2004) }\end{array}$ & Free Surface & Circular & $\mathrm{Nu}=0.798 \operatorname{Re}^{1 / 2} \operatorname{Pr}^{1 / 2}\left(\frac{\mathrm{d}}{\mathrm{H}}\right)^{1 / 2}$ & Not mentioned & Experimental & - \\
\hline
\end{tabular}


Continuation Table 1

\begin{tabular}{|c|c|c|c|c|c|c|}
\hline Ref. No. & Type of jet & $\begin{array}{l}\text { Nozzle } \\
\text { geometry }\end{array}$ & Nusselt Correlation & Reynolds range & Type of Study & Notes \\
\hline $\begin{array}{l}\text { Garimella and } \\
\text { Rice (1995) }\end{array}$ & $\begin{array}{l}\text { Confined } \\
\text { Submerged }\end{array}$ & Circular & $\mathrm{Nu}=0.160 \operatorname{Re}^{0.695} \operatorname{Pr}^{0.4}\left(\frac{\mathrm{S}_{\mathrm{NP}}}{\mathrm{d}}\right)^{-0.11}\left(\frac{\mathrm{l}_{\mathrm{N}}}{\mathrm{d}}\right)^{-0.11}$ & $4000<\operatorname{Re}<23000$ & Experimental & $\begin{array}{l}1.59 \leq \mathrm{d} \leq 6.35 \mathrm{~mm} \\
1 \leq \mathrm{S}_{\mathrm{NP}} / \mathrm{d} \leq 5 \\
0.25 \leq 1_{\mathrm{N}} / \mathrm{d} \leq 12\end{array}$ \\
\hline $\begin{array}{l}\text { Garimella and } \\
\text { Rice (1995) }\end{array}$ & $\begin{array}{l}\text { Confined } \\
\text { Submerged }\end{array}$ & Circular & $\mathrm{Nu}=0.164 \operatorname{Re}^{0.773} \operatorname{Pr}^{0.4}\left(\frac{\mathrm{S}_{\mathrm{NP}}}{\mathrm{d}}\right)^{-0.52}\left(\frac{\mathrm{l}_{\mathrm{N}}}{\mathrm{d}}\right)^{-0.05}$ & $4000<\operatorname{Re}<23000$ & Experimental & $\begin{array}{l}1.59 \leq \mathrm{d} \leq 6.35 \mathrm{~mm} \\
6 \leq \mathrm{S}_{\mathrm{NP}} / \mathrm{d} \leq 14 \\
0.25 \leq 1_{\mathrm{N}} / \mathrm{d} \leq 12\end{array}$ \\
\hline $\begin{array}{l}\text { Womac et al. } \\
\text { (1996) }\end{array}$ & Submerged & Circular & $\begin{array}{l}\frac{\mathrm{Nu}}{\operatorname{Pr}^{0.4}}=\mathrm{C}_{1} \operatorname{Re}_{\mathrm{d}}^{\mathrm{m}} \frac{1}{\mathrm{~d}} \mathrm{~A}_{\mathrm{r}}+\mathrm{C}_{2} \operatorname{Re}_{\mathrm{L}}^{\mathrm{n}} \frac{1}{\mathrm{~L}}\left(1-\mathrm{A}_{\mathrm{r}}\right) \\
\mathrm{L}=\frac{\left[\left(\sqrt{2} \mathrm{~L}_{\mathrm{e}} / 2\right)-1.9 \mathrm{~d}\right]+\left[\left(\mathrm{L}_{\mathrm{e}} / 2\right)-1.9 \mathrm{~d}\right]}{2} \\
\mathrm{~A}_{\mathrm{r}}=\mathrm{N} \pi(1.9 \mathrm{~d})^{2} / 1^{2}\end{array}$ & $5000<\operatorname{Re}<200000$ & Experimental & $\begin{array}{l}\text { Multiple jet } \\
0.5 \leq \mathrm{d} \leq 1.0 \mathrm{~mm} \\
2 \leq \mathrm{S}_{\mathrm{NP}} / \mathrm{d} \leq 4 \\
\mathrm{~N}=4 \text { or } 9 \text { jets } \\
1=127 \mathrm{~mm} \\
\mathrm{n}=0.8, \quad \mathrm{C}_{1}=0.509 \\
\mathrm{C}_{2}=0.0363\end{array}$ \\
\hline $\begin{array}{l}\text { Churchill and } \\
\text { Bernstein } \\
\text { (1977) }\end{array}$ & Confined & Circular & $\overline{\mathrm{Nu}}=0.3+\frac{0.62 \mathrm{Re}^{1 / 2} \operatorname{Pr}^{1 / 3}}{\left[1+(0.4 / \mathrm{Pr})^{2 / 3}\right]^{1 / 4}} \times\left[1+\left(\frac{\mathrm{Re}}{282000}\right)^{5 / 8}\right]^{4 / 5}$ & Not mentioned & Numerical & $\begin{array}{l}\text { convection from gases and } \\
\text { liquids to a circular cylinder } \\
\text { in crossflow }\end{array}$ \\
\hline $\begin{array}{l}\text { Zhang and } \mathrm{Hu} \\
(2005)\end{array}$ & Confined & Circular & $\mathrm{Nu}_{\mathrm{o}}=1.215 \operatorname{Re}^{0.4884} \operatorname{Pr}^{1 / 3}(\mathrm{~L} / \mathrm{D})^{-0.0043}$ & Not mentioned & Experimental & Target: curved surface \\
\hline $\begin{array}{l}\text { Sun et al. } \\
(1998)\end{array}$ & Free Surface & Circular & $\begin{array}{l}\mathrm{Nu} / \mathrm{Nu}_{\mathrm{o}}=0.777(\mathrm{r} / \mathrm{d})^{-0.5} \text { for } 1<\mathrm{r} / \mathrm{d}<\mathrm{r}_{\mathrm{v}} / \mathrm{d} \\
\mathrm{Nu} / \mathrm{Nu}_{\mathrm{o}}=1.85 \operatorname{Re}^{-1 / 6}\left[\frac{25.7}{\operatorname{Re}}(\mathrm{r} / \mathrm{d})^{3}+0.857\right]^{-2 / 3} \\
: \mathrm{r} / \mathrm{d}>\mathrm{r}_{\mathrm{v}} / \mathrm{d}, \mathrm{r}_{\mathrm{v}} / \mathrm{d}=0.177 \operatorname{Re}\end{array}$ & $\operatorname{Re}<100000$ & Experimental & - \\
\hline $\begin{array}{l}\text { Lallave Cortes } \\
(2009)\end{array}$ & Free Surface & Circular & $\mathrm{Nu}_{\mathrm{av}}=\mathrm{Re}^{0.385} \cdot \mathrm{Ek}^{-0.091} \cdot \operatorname{Pr}^{0.4} \cdot \beta^{0.0114} \cdot \varepsilon^{-0.25}$ & $445<\operatorname{Re}<1800$ & Numerical & $\begin{array}{l}\text { Spinning target } \\
2.2 \times 10^{-5} \leq \mathrm{Ek} \leq 2.65 \times 10^{-4} \\
\operatorname{Pr}=5.49, \quad 0.55 \leq \beta \leq 5.0 \\
227.6 \leq \varepsilon \leq 697.5\end{array}$ \\
\hline $\begin{array}{l}\text { Lallave Cortes } \\
(2009)\end{array}$ & Free Surface & Circular & $\mathrm{Nu}_{\mathrm{av}}=1.965 \mathrm{Re}^{0.3875} \cdot \mathrm{Ek}^{-0.091} \cdot \varepsilon^{-0.25} \cdot \mathrm{Fo}^{0.01}$ & $500<\operatorname{Re}<110$ & Numerical & $\begin{array}{l}6.62 \times 10^{-5} \leq \mathrm{Ek} \leq 2.65 \times 10^{-4} \\
=2.67, \quad \operatorname{Pr}=5.49 \\
227.6 \leq \varepsilon \leq 697.5 \\
0.031 \leq \mathrm{Fo} \leq 0.504\end{array}$ \\
\hline $\begin{array}{l}\text { Lallave Cortes } \\
(2009)\end{array}$ & Confined & Circular & $\mathrm{Nu}_{\mathrm{av}}=\mathrm{Re}^{1.26} \cdot \mathrm{Ek}^{-0.1111} \cdot \mathrm{Pr}^{-2.58} \cdot \beta^{-0.5} \cdot \varepsilon^{-0.65}$ & $750<\operatorname{Re}<2000$ & Numerical & $\begin{array}{l}\text { Spinning target, } \\
6.62 \times 10^{-5} \leq \mathrm{Ek} \leq 2.65 \times 10^{-4} \\
\operatorname{Pr}=5.49, \quad 0.55 \leq \beta \leq 5.0 \\
227.6 \leq \varepsilon \leq 697.5\end{array}$ \\
\hline $\begin{array}{l}\text { Lallave Cortes } \\
(2009)\end{array}$ & $\begin{array}{l}\text { Spinning } \\
\text { Confined }\end{array}$ & Circular & $\begin{aligned} \mathrm{Nu}_{\mathrm{av}} & =1.9762 \cdot \beta^{-0.01} \cdot \mathrm{Re}^{0.75} \\
& . \mathrm{Ek}^{-0.111} \cdot\left(\mathrm{b} / \mathrm{d}_{\mathrm{n}}\right)^{-0.05} \cdot \varepsilon^{-0.69}\end{aligned}$ & $500<\operatorname{Re}<1500$ & Numerical & $\begin{array}{l}\text { Stationary Target, } \\
4.25 \times 10^{-5} \leq \mathrm{Ek} \leq 1.06 \times 10^{-4} \\
0.25 \leq \beta \leq 5, \quad \operatorname{Pr}=5.49 \\
0.25 \leq \mathrm{b} / \mathrm{d}_{\mathrm{n}} \leq 1.67 \\
227.6 \leq \varepsilon \leq 627.6\end{array}$ \\
\hline $\begin{array}{l}\text { Lallave Cortes } \\
(2009)\end{array}$ & $\begin{array}{l}\text { Stationary } \\
\text { Partially } \\
\text { Confined }\end{array}$ & Circular & $\begin{aligned} \mathrm{Nu}_{\mathrm{av}}=1.94282 \beta^{0.1} \cdot \mathrm{Re}^{0.75} \\
. \mathrm{Ek}^{-0.1} \cdot \varepsilon^{-0.7} \cdot\left(\mathrm{r}_{\mathrm{p}} / \mathrm{r}_{\mathrm{d}}\right)^{-0.05}\end{aligned}$ & $360<\operatorname{Re}<900$ & Numerical & $\begin{array}{l}\text { Spinning Target, } \\
1.06 \times 10^{-4} \leq \mathrm{Ek} \leq 4.25 \times 10^{-4} \\
0.25 \leq \beta \leq 1, \quad \operatorname{Pr}=5.49 \\
0.2 \leq \mathrm{r}_{\mathrm{p}} / \mathrm{r}_{\mathrm{d}} \leq 0.75 \\
227.6 \leq \varepsilon \leq 627.6\end{array}$ \\
\hline $\begin{array}{l}\text { Lallave Cortes } \\
(2009)\end{array}$ & $\begin{array}{l}\text { Spinning } \\
\text { Confined }\end{array}$ & Circular & $\begin{aligned} \mathrm{Nu}_{\mathrm{av}}= & 1.94282 \beta^{-0.01} \cdot \mathrm{Re}^{0.75} \cdot \mathrm{Ek}_{1}{ }^{-0.0465} \\
& . \mathrm{Ek}_{2}{ }^{-0.047} . \varepsilon^{-0.69} \cdot\left(\mathrm{r}_{\mathrm{p}} / \mathrm{r}_{\mathrm{d}}\right)^{-0.05}\end{aligned}$ & $360<\operatorname{Re}<900$ & Numerical & $\begin{array}{l}\text { Spinning Target, } \\
4.25 \times 10^{-4} \leq \mathrm{Ek}_{1} \leq 7.08 \times 10^{-5} \\
4.25 \times 10^{-4} \leq \mathrm{Ek}_{2} \leq 7.08 \times 10^{-5} \\
0.25 \leq \beta \leq 1, \quad \operatorname{Pr}=5.49 \\
0.2 \leq \mathrm{r}_{\mathrm{p}} / \mathrm{r}_{\mathrm{d}} \leq 0.75 \\
227.6 \leq \varepsilon \leq 627.6\end{array}$ \\
\hline
\end{tabular}

Continuation Table 1 


\section{Continuation Table 1}

\begin{tabular}{|c|c|c|c|c|c|c|}
\hline Ref. No. & Type of jet & $\begin{array}{c}\text { Nozzle } \\
\text { geometry }\end{array}$ & Nusselt Correlation & Reynolds range & Type of Study & Notes \\
\hline $\begin{array}{l}\text { Lallave Cortes } \\
\text { (2009) }\end{array}$ & $\begin{array}{l}\text { Partially } \\
\text { Confined }\end{array}$ & Circular & $\begin{aligned} \mathrm{Nu}_{\mathrm{av}}= & 1.94282 \beta^{-0.01} \cdot \mathrm{Re}^{0.74} \cdot \mathrm{Ek}^{-0.1} \\
& .\left(\mathrm{b} / \mathrm{d}_{\mathrm{n}}\right)^{-0.05} \cdot\left(\mathrm{r}_{\mathrm{p}} / \mathrm{r}_{\mathrm{d}}\right)^{-0.05} \cdot \mathrm{Fo}^{-0.01}\end{aligned}$ & $225<\operatorname{Re}<900$ & Numerical & $\begin{array}{l}\text { Spinning Target, } \\
7.08 \times 10^{-5} \leq \mathrm{Ek} \leq 4.25 \times 10^{-4} \\
0.25 \leq \beta \leq 1, \quad \operatorname{Pr}=5.49 \\
0.2 \leq \mathrm{r}_{\mathrm{p}} / \mathrm{r}_{\mathrm{d}} \leq 0.75 \\
0.2 \leq \mathrm{r}_{\mathrm{p}} / \mathrm{r}_{\mathrm{d}} \leq 0.75, \\
0.045 \leq \mathrm{Fo} \leq 0.72 \\
227.6 \leq \varepsilon \leq 376.7\end{array}$ \\
\hline $\begin{array}{l}\text { Fujii and Fujii } \\
\text { (1976) }\end{array}$ & Confined & Circular & $\mathrm{Nu}_{\mathrm{c}, \mathrm{s}}=\left(\frac{\mathrm{Pr}}{4+9 \mathrm{Pr}^{1 / 2}+10 \operatorname{Pr}}\right)^{1 / 5}\left(\mathrm{Gr}_{\mathrm{c}}^{*} \operatorname{Pr}\right)^{1 / 5}$ & Not mentioned & Numerical & - \\
\hline $\begin{array}{l}\text { Li and } \\
\text { Garimella } \\
(2001)\end{array}$ & $\begin{array}{l}\text { Confined } \\
\text { Submerged }\end{array}$ & Circular & $\begin{aligned} \mathrm{Nu}_{\mathrm{av}}= & 1.064 \mathrm{Re}^{0.513} \operatorname{Pr}^{0.441}\left(\frac{1}{\mathrm{~d}}\right)^{-0.071}\left(\frac{\mathrm{D}_{\mathrm{e}}}{\mathrm{d}}\right)^{-0.266} \cdot \mathrm{A}_{\mathrm{r}} \\
& +1.291 \mathrm{Re}^{0.63} \operatorname{Pr}^{0.441}\left(\frac{\mathrm{D}_{\mathrm{e}}}{\mathrm{d}}\right)^{-1.063} \cdot\left(1-\mathrm{A}_{\mathrm{r}}\right)\end{aligned}$ & $4000<\operatorname{Re}<23000$ & Experimental & $\begin{array}{l}1.59 \mathrm{~mm} \leq \mathrm{d} \leq 6.35 \\
7.1 \leq \operatorname{Pr} \leq 25.2 \\
1 \leq \mathrm{H} / \mathrm{d} \leq 5 \\
0.25 \leq 1 / \mathrm{d} \leq 12 \\
\mathrm{D}_{\mathrm{e}}=11.28 \mathrm{~mm}\end{array}$ \\
\hline $\begin{array}{l}\text { Sung and } \\
\text { Mudawar } \\
\text { (2008) }\end{array}$ & Free Surface & Circular & 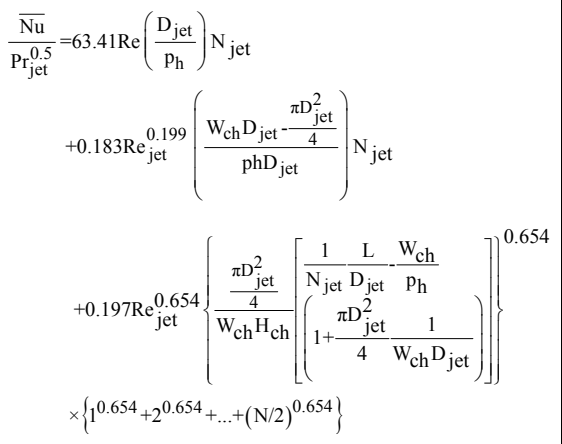 & $1000<\operatorname{Re}<3500$ & $\begin{array}{l}\text { Experimental } \\
\text { and } \\
\text { Numerical }\end{array}$ & $\begin{array}{l}\text { Hybrid cooling with N-nozzles: } \\
\text { micro-channel flow and micro- } \\
\text { jet impingement }\end{array}$ \\
\hline $\begin{array}{l}\text { Chen } e t a l \text {. } \\
\text { (2005) }\end{array}$ & Free Surface & Slot & $\mathrm{Nu}=\left[\frac{2 \varepsilon}{\operatorname{PrRe}}+\frac{272 \mathrm{H}}{525 \mathrm{~B}}\right]^{-1}, \mathrm{H}=3.858 \frac{\mathrm{x}}{\operatorname{Re}}+\left(0.8-\frac{0.6277}{\operatorname{Re}^{0.305}}\right)$ & $\operatorname{Re}<100000$ & Theoretical & - \\
\hline $\begin{array}{l}\text { Teameh and } \\
\text { Farahat } \\
\text { (2003) }\end{array}$ & Free Surface & Circular & $\overline{\mathrm{Nu}}=10^{-2.25223}(\mathrm{r} / \mathrm{d})^{-1.15607} \mathrm{Re}^{1.1528288}$ & $25000<\operatorname{Re}<40000$ & $\begin{array}{l}\text { Experimental } \\
\text { and } \\
\text { Numerical }\end{array}$ & $3.6<\mathrm{r} / \mathrm{d}<54$ \\
\hline $\begin{array}{l}\text { Teameh and } \\
\text { Farahat } \\
\text { (2003) }\end{array}$ & Free Surface & Circular & $\overline{\mathrm{Nu}}=10^{0.755395}(\mathrm{r} / \mathrm{d})^{-1.50812} \mathrm{Re}^{0.56188}$ & $5000<\operatorname{Re}<20000$ & $\begin{array}{l}\text { Experimental } \\
\text { and } \\
\text { Numerical }\end{array}$ & $3.6<\mathrm{r} / \mathrm{d}<54$ \\
\hline $\begin{array}{l}\text { Stevens et al. } \\
\text { (1991) }\end{array}$ & Free surface & Circular & $\mathrm{Nu}=0.93 \operatorname{Re}_{\mathrm{d}}^{1 / 2} \operatorname{Pr}^{0.4}$ & Not mentioned & Numerical & Turbulent flow \\
\hline $\begin{array}{l}\text { Rahman et al. } \\
\text { (2008) }\end{array}$ & Semi-confined & Circular & $\begin{aligned} \overline{\mathrm{Nu}}= & 1.94282 \beta^{0.111} \mathrm{Re}^{0.75} \mathrm{Ek}_{1}^{-0.0465} \mathrm{Ek}_{2}^{-0.047} . \\
& . \varepsilon^{-0.69}\left(\mathrm{r}_{\mathrm{p}} / \mathrm{r}_{\mathrm{d}}\right)^{-0.05}\end{aligned}$ & $220<\operatorname{Re}<900$ & Numerical & $\begin{array}{l}\text { uniformly heated spinning } \\
\text { solid disk; rotating nozzle }\end{array}$ \\
\hline $\begin{array}{l}\text { Robinson and } \\
\text { Schnitzler } \\
\text { (2007) }\end{array}$ & $\begin{array}{l}\text { Free and } \\
\text { Submerged }\end{array}$ & Circular & $\begin{array}{l}\frac{\mathrm{Nu}}{\operatorname{Pr}^{0.4}}=7.8 \operatorname{Re}_{d_{n}}^{0.49} \exp \left(-0.025 \frac{\mathrm{S}}{\mathrm{d}_{\mathrm{n}}}\right) \\
\frac{\mathrm{Nu}}{\operatorname{Pr}^{0.4}}=23.39 \operatorname{Re}_{\mathrm{d}_{\mathrm{n}}}^{0.46}\left(\frac{\mathrm{s}}{\mathrm{d}_{\mathrm{n}}}\right)^{-0.442}\left(\frac{\mathrm{H}}{\mathrm{d}_{\mathrm{n}}}\right)^{-0.00716}\end{array}$ & $100<\operatorname{Re}<10000$ & Experimental & $\begin{array}{l}\text { Miniature jet array } \\
2 \leq \mathrm{H} / \mathrm{dn} \leq 3\end{array}$ \\
\hline $\begin{array}{l}\text { Jiji and Dagan } \\
\text { (1987) }\end{array}$ & Free surface & Circular & $\overline{\mathrm{Nu}}=3.84 \mathrm{Re}^{1 / 2} \operatorname{Pr}^{1 / 3}\left[0.008 \frac{\mathrm{Ln}}{\mathrm{d}}+1\right]$ & Not mentioned & Experimental & Multijet \\
\hline $\begin{array}{l}\text { Pan and Webb } \\
\text { (1993) }\end{array}$ & Free surface & Circular & $\overline{\mathrm{Nu}}=0.225 \operatorname{Re}^{2 / 3} \operatorname{Pr}^{1 / 3} \mathrm{e}^{-0.095(\mathrm{~s} / \mathrm{d})}$ & Not mentioned & Numerical & arrays of free-surface jets \\
\hline $\begin{array}{l}\text { Zuckerman } \\
\text { and Lior } \\
\text { (2007) }\end{array}$ & Free surface & Slot & $\mathrm{Nu}_{\mathrm{av}}=0.12\left(\frac{\mathrm{d}}{\mathrm{D}}\right)^{-0.16}(\mathrm{n})^{0.18}(\mathrm{Re})^{0.66}(\mathrm{Pr})^{0.5}$ & $5000<\operatorname{Re}<80000$ & Numerical & $\begin{array}{l}2 \leq \mathrm{n} \leq 8 \\
0.7 \leq \operatorname{Pr} \leq 5.85 \\
2 \leq \mathrm{H} / 2 \mathrm{~B} \leq 5\end{array}$ \\
\hline
\end{tabular}

Continuation Table 1 


\section{Continuation Table 1}

\begin{tabular}{|c|c|c|c|c|c|c|}
\hline Ref. No. & Type of jet & $\begin{array}{l}\text { Nozzle } \\
\text { geometry }\end{array}$ & Nusselt Correlation & Reynolds range & Type of Study & Notes \\
\hline $\begin{array}{l}\text { Patel and Roy } \\
\text { (2001) }\end{array}$ & Free surface & Rectangular & $\begin{array}{l}\mathrm{Nu}=-\left(0.0436 \mathrm{Re}^{2}+0.021 \mathrm{Re}+0.0081\right) \theta^{2} \\
\left(5.5 \mathrm{Re}^{2}+2.77 \operatorname{Re}+2.45\right) \theta \\
(103.2 \operatorname{Re}+140.4-130.8)\end{array}$ & $5000 \leq \operatorname{Re} \leq 20000$ & Numerical & $\begin{array}{l}\text { Jet angle } 30^{\circ} \leq \theta \leq 90^{\circ} \\
\text { a Pair of Rectangular Jets }\end{array}$ \\
\hline $\begin{array}{l}\text { Lallave } e t a l \text {. } \\
\text { (2007) }\end{array}$ & Confined & Circular & $\overline{\mathrm{Nu}}=1.97619 \beta^{0.0909} \mathrm{Re}^{0.75} \mathrm{Ek}^{-0.1111} \varepsilon^{-0.9}$ & $500<\operatorname{Re}<1500$ & Numerical & Target: rotating disk \\
\hline $\begin{array}{l}\text { Fang and } \\
\text { Kasagi (2007) }\end{array}$ & Free Surface & Circular & $\mathrm{Nu}_{\mathrm{o}}=0.409 \mathrm{Re}^{0.559} \operatorname{Pr}^{1 / 3}$ & $849<\operatorname{Re}<2124$ & Experimental & Micro-scale jet \\
\hline $\begin{array}{l}\text { Liu and Gabour } \\
\text { (1993) }\end{array}$ & Confined & Circular & $\mathrm{Nu}_{\mathrm{o}}=0.797 \operatorname{Re}^{1 / 2} \operatorname{Pr}^{1 / 3}$ & Not mentioned & Numerical & laminar jets \\
\hline $\begin{array}{l}\text { Wolf et al. } \\
(1995)\end{array}$ & Free Surface & Circular & $\mathrm{Nu}_{\mathrm{o}}=0.202 \mathrm{Re}^{0.620} \operatorname{Pr}^{0.4}$ & Not mentioned & Experimental & $\begin{array}{l}\text { Turbulence dissipation in a } \\
\text { free surface jet of water }\end{array}$ \\
\hline $\begin{array}{l}\text { Vader } \text { et al. } \\
\text { (1991) }\end{array}$ & Confined & Planar & $\mathrm{Nu}_{\mathrm{o}}=0.28 \operatorname{Re}^{0.58} \operatorname{Pr}^{0.4}$ & $20000<\operatorname{Re}<90000$ & Numerical & $2.7<\operatorname{Pr}<4.5$ \\
\hline $\begin{array}{l}\text { Womac et al. } \\
\text { (1993) }\end{array}$ & Submerged & Circular & $\begin{array}{l}\frac{\mathrm{Nu}}{\operatorname{Pr}^{0.4}}=\mathrm{C}_{1} \operatorname{Re}_{\mathrm{d}}^{\mathrm{m}} \frac{1}{\mathrm{~d}} \mathrm{~A}_{\mathrm{r}}+\mathrm{C}_{2} \operatorname{Re}_{\mathrm{L}}^{\mathrm{n}} \frac{1}{\mathrm{~L}}\left(1-\mathrm{A}_{\mathrm{r}}\right) \\
\mathrm{L}=\frac{(0.5 \sqrt{2} 1-1.9 \mathrm{~d})+(0.51-1.9 \mathrm{~d})}{2} \mathrm{~A}_{\mathrm{r}}=\pi(1.9 \mathrm{~d})^{2} / 1^{2}\end{array}$ & $\operatorname{Re}<50000$ & Numerical & $1.65 \leq \mathrm{d} \leq 6.55 \mathrm{~mm}$ \\
\hline $\begin{array}{l}\text { Womac et al. } \\
\text { (1993) }\end{array}$ & Free surface & Circular & $\begin{array}{l}\frac{\mathrm{Nu}}{\operatorname{Pr}^{0.4}}=\mathrm{C}_{1} \operatorname{Re}_{\mathrm{d}_{\mathrm{i}}} \frac{\mathrm{l}}{\mathrm{d}_{\mathrm{i}}} \mathrm{A}_{\mathrm{r}}+\mathrm{C}_{2} \operatorname{Re}_{\mathrm{L}}^{\mathrm{n}} \frac{1}{\mathrm{~L}}\left(1-\mathrm{A}_{\mathrm{r}}\right) \\
\mathrm{L}=\frac{\left(0.5 \sqrt{2} 1-\mathrm{d}_{\mathrm{i}}\right)+\left(0.51-\mathrm{d}_{\mathrm{i}}\right)}{2} \mathrm{~A}_{\mathrm{r}}=\pi \mathrm{d}_{\mathrm{i}}^{2} / 1^{2}\end{array}$ & $\operatorname{Re}<50000$ & Experimental & $\begin{array}{l}1.65 \leq \mathrm{d} \leq 6.55 \mathrm{~mm} \\
3.5 \leq \mathrm{S}_{\mathrm{NP}} / \mathrm{d} \leq 10 \\
\mathrm{l}=12.7 \mathrm{~mm}, \mathrm{~m}=0.5 \\
\mathrm{n}=0.532, \mathrm{C}_{1}=0.516 \\
\mathrm{C}_{2}=0.491\end{array}$ \\
\hline $\begin{array}{l}\text { Silverman and } \\
\text { Nagler (2004) }\end{array}$ & Free Surface & Circular & $\mathrm{Nu}=0.798 \operatorname{Re}^{1 / 2} \operatorname{Pr}^{1 / 2}\left(\frac{\mathrm{d}}{\mathrm{H}}\right)^{1 / 2}$ & Not mentioned & Experimental & - \\
\hline $\begin{array}{l}\text { Garimella and } \\
\text { Rice (1995) }\end{array}$ & $\begin{array}{l}\text { Confined } \\
\text { Submerged }\end{array}$ & Circular & $\mathrm{Nu}=0.160 \operatorname{Re}^{0.695} \operatorname{Pr}^{0.4}\left(\frac{\mathrm{S}_{\mathrm{NP}}}{\mathrm{d}}\right)^{-0.11}\left(\frac{1_{\mathrm{N}}}{\mathrm{d}}\right)^{-0.11}$ & $4000<\operatorname{Re}<23000$ & Experimental & $\begin{array}{l}1.59 \leq \mathrm{d} \leq 6.35 \mathrm{~mm} \\
1 \leq \mathrm{S}_{\mathrm{NP}} / \mathrm{d} \leq 5 \\
0.25 \leq \mathrm{1}_{\mathrm{N}} / \mathrm{d} \leq 12\end{array}$ \\
\hline $\begin{array}{l}\text { Garimella and } \\
\text { Rice (1995) }\end{array}$ & $\begin{array}{l}\text { Confined } \\
\text { Submerged }\end{array}$ & Circular & $\mathrm{Nu}=0.164 \operatorname{Re}^{0.773} \operatorname{Pr}^{0.4}\left(\frac{\mathrm{S}_{\mathrm{NP}}}{\mathrm{d}}\right)^{-0.52}\left(\frac{\mathrm{l}_{\mathrm{N}}}{\mathrm{d}}\right)^{-0.05}$ & $4000<\operatorname{Re}<23000$ & Experimental & $\begin{array}{l}1.59 \leq \mathrm{d} \leq 6.35 \mathrm{~mm} \\
1 \leq \mathrm{S}_{\mathrm{NP}} / \mathrm{d} \leq 5 \\
0.25 \leq \mathrm{l}_{\mathrm{N}} / \mathrm{d} \leq 12\end{array}$ \\
\hline $\begin{array}{l}\text { Womac et al. } \\
\text { (1996) }\end{array}$ & Submerged & Circular & $\begin{array}{l}\frac{\mathrm{Nu}}{\operatorname{Pr}^{0.4}}=\mathrm{C}_{1} \operatorname{Re}_{\mathrm{d}}^{\mathrm{m}} \frac{1}{\mathrm{~d}} \mathrm{~A}_{\mathrm{r}}+\mathrm{C}_{2} \operatorname{Re}_{\mathrm{L}}^{\mathrm{n}} \frac{1}{\mathrm{~L}}\left(1-\mathrm{A}_{\mathrm{r}}\right) \\
\mathrm{L}=\frac{\left[\left(\sqrt{2} \mathrm{~L}_{\mathrm{e}} / 2\right)-1.9 \mathrm{~d}\right]+\left[\left(\mathrm{L}_{\mathrm{e}} / 2\right)-1.9 \mathrm{~d}\right]}{2} \\
\mathrm{~A}_{\mathrm{r}}=\mathrm{N} \pi(1.9 \mathrm{~d})^{2} / 1^{2}\end{array}$ & $5000<\operatorname{Re}<200000$ & Experimental & $\begin{array}{l}\text { Multiple jet } \\
0.5 \leq \mathrm{d} \leq 1.0 \mathrm{~mm} \\
2 \leq \mathrm{S}_{\mathrm{NP}} / \mathrm{d} \leq 4 \\
\mathrm{~N}=4 \text { or } 9 \text { jets } \\
1=127 \mathrm{~mm} \\
\mathrm{n}=0.8, \quad \mathrm{C}_{1}=0.509 \\
\mathrm{C}_{2}=0.0363\end{array}$ \\
\hline $\begin{array}{l}\text { Churchill and } \\
\text { Bernstein } \\
\text { (1977) }\end{array}$ & Confined & Circular & $\overline{\mathrm{Nu}}=0.3+\frac{0.62 \mathrm{Re}^{1 / 2} \operatorname{Pr}^{1 / 3}}{\left[1+(0.4 / \mathrm{Pr})^{2 / 3}\right]^{1 / 4}} \times\left[1+\left(\frac{\mathrm{Re}}{282000}\right)^{5 / 8}\right]^{4 / 5}$ & Not mentioned & Numerical & $\begin{array}{l}\text { convection from gases and } \\
\text { liquids to a circular cylinder } \\
\text { in crossflow }\end{array}$ \\
\hline $\begin{array}{l}\text { Zhang and } \mathrm{Hu} \\
(2005)\end{array}$ & Confined & Circular & $\mathrm{Nu}_{\mathrm{o}}=1.215 \operatorname{Re}^{0.4884} \operatorname{Pr}^{1 / 3}(\mathrm{~L} / \mathrm{D})^{-0.0043}$ & Not mentioned & Experimental & Target: curved surface \\
\hline $\begin{array}{l}\text { Sun et al. } \\
(1998)\end{array}$ & Free Surface & Circular & $\begin{array}{l}\mathrm{Nu} / \mathrm{Nu}_{\mathrm{o}}=0.777(\mathrm{r} / \mathrm{d})^{-0.5} \text { for } 1<\mathrm{r} / \mathrm{d}<\mathrm{r}_{\mathrm{v}} / \mathrm{d} \\
\mathrm{Nu} / \mathrm{Nu}_{\mathrm{o}}=1.85 \operatorname{Re}^{-1 / 6}\left[\frac{25.7}{\operatorname{Re}}(\mathrm{r} / \mathrm{d})^{3}+0.857\right]^{-2 / 3} \\
: \mathrm{r} / \mathrm{d}>\mathrm{r}_{\mathrm{v}} / \mathrm{d}, \mathrm{r}_{\mathrm{v}} / \mathrm{d}=0.177 \operatorname{Re}\end{array}$ & $\operatorname{Re}<100000$ & Experimental & - \\
\hline $\begin{array}{l}\text { Lallave Cortes } \\
(2009)\end{array}$ & Free Surface & Circular & $\mathrm{Nu}_{\mathrm{av}}=\mathrm{Re}^{0.385} \cdot \mathrm{Ek}^{-0.091} \cdot \operatorname{Pr}^{0.4} \cdot \beta^{0.0114} \cdot \varepsilon^{-0.25}$ & $445<\operatorname{Re}<1800$ & Numerical & $\begin{array}{l}\text { Spinning Target, } \\
2.2 \times 10^{-5} \leq \mathrm{Ek} \leq 2.65 \times 10^{-4} \\
\operatorname{Pr}=5.49, \quad 0.55 \leq \beta \leq 5.0 \\
227.6 \leq \varepsilon \leq 697.5\end{array}$ \\
\hline
\end{tabular}

Continuation Table 1 


\section{Continuation Table 1}

\begin{tabular}{|c|c|c|c|c|c|c|}
\hline Ref. No. & Type of jet & $\begin{array}{l}\text { Nozzle } \\
\text { geometry }\end{array}$ & Nusselt Correlation & Reynolds range & Type of Study & Notes \\
\hline $\begin{array}{l}\text { Lallave Cortes } \\
(2009)\end{array}$ & Free Surface & Circular & $\mathrm{Nu}_{\mathrm{av}}=1.965 \mathrm{Re}^{0.3875} \cdot \mathrm{Ek}^{-0.091} \cdot \varepsilon^{-0.25} \cdot \mathrm{Fo}^{0.01}$ & $500<\operatorname{Re}<110$ & Numerical & $\begin{array}{l}6.62 \times 10^{-5} \leq \mathrm{Ek} \leq 2.65 \times 10^{-4} \\
=2.67, \quad \operatorname{Pr}=5.49 \\
227.6 \leq \varepsilon \leq 697.5 \\
0.031 \leq \mathrm{Fo} \leq 0.504\end{array}$ \\
\hline $\begin{array}{l}\text { Lallave Cortes } \\
(2009)\end{array}$ & Confined & Circular & $\mathrm{Nu}_{\mathrm{av}}=\operatorname{Re}^{1.26} \cdot \mathrm{Ek}^{-0.1111} \cdot \operatorname{Pr}^{-2.58} \cdot \beta^{-0.5} \cdot \varepsilon^{-0.65}$ & $750<\operatorname{Re}<2000$ & Numerical & $\begin{array}{l}\text { Spinning Target, } \\
6.62 \times 10^{-5} \leq \mathrm{Ek} \leq 2.65 \times 10^{-4} \\
\operatorname{Pr}=5.49, \quad 0.55 \leq \beta \leq 5.0 \\
227.6 \leq \varepsilon \leq 697.5\end{array}$ \\
\hline $\begin{array}{l}\text { Lallave Cortes } \\
(2009)\end{array}$ & $\begin{array}{l}\text { Spinning } \\
\text { Confined }\end{array}$ & Circular & $\begin{aligned} \mathrm{Nu}_{\mathrm{av}}= & 1.9762 . \beta^{-0.01} \cdot \mathrm{Re}^{0.75} \cdot \mathrm{Ek}^{-0.111} \\
& .\left(\mathrm{b} / \mathrm{d}_{\mathrm{n}}\right)^{-0.05} \cdot \varepsilon^{-0.69}\end{aligned}$ & $500<\operatorname{Re}<1500$ & Numerical & $\begin{array}{l}\text { Stationary Target, } \\
4.25 \times 10^{-5} \leq \mathrm{Ek} \leq 1.06 \times 10^{-4} \\
0.25 \leq \beta \leq 5, \quad \operatorname{Pr}=5.49 \\
0.25 \leq \mathrm{b} / \mathrm{d}_{\mathrm{n}} \leq 1.67 \\
227.6 \leq \varepsilon \leq 627.6\end{array}$ \\
\hline $\begin{array}{l}\text { Lallave Cortes } \\
(2009)\end{array}$ & $\begin{array}{l}\text { Stationary } \\
\text { Partially } \\
\text { Confined }\end{array}$ & Circular & $\begin{aligned} \mathrm{Nu}_{\mathrm{av}}= & 1.94282 \beta^{0.1} \cdot \mathrm{Re}^{0.75} \cdot \mathrm{Ek}^{-0.1} \\
& . \varepsilon^{-0.7} \cdot\left(\mathrm{r}_{\mathrm{p}} / \mathrm{r}_{\mathrm{d}}\right)^{-0.05}\end{aligned}$ & $360<\operatorname{Re}<900$ & Numerical & $\begin{array}{l}\text { Spinning Target, } \\
1.06 \times 10^{-4} \leq \mathrm{Ek} \leq 4.25 \times 10^{-4} \\
0.25 \leq \beta \leq 1, \quad \operatorname{Pr}=5.49 \\
0.2 \leq \mathrm{r}_{\mathrm{p}} / \mathrm{r}_{\mathrm{d}} \leq 0.75 \\
227.6 \leq \varepsilon \leq 627.6\end{array}$ \\
\hline $\begin{array}{l}\text { Lallave Cortes } \\
(2009)\end{array}$ & $\begin{array}{l}\text { Spinning } \\
\text { Confined }\end{array}$ & Circular & $\begin{aligned} \mathrm{Nu}_{\mathrm{av}}= & 1.94282 \beta^{-0.01} \cdot \operatorname{Re}^{0.75} \cdot \mathrm{Ek}_{1}{ }^{-0.0465} \\
& . \mathrm{Ek}_{2}{ }^{-0.047} \cdot \varepsilon^{-0.69} \cdot\left(\mathrm{r}_{\mathrm{p}} / \mathrm{r}_{\mathrm{d}}\right)^{-0.05}\end{aligned}$ & $360<\operatorname{Re}<900$ & Numerical & $\begin{array}{l}\text { Spinning Target, } \\
4.25 \times 10^{-4} \leq \mathrm{Ek}_{1} \leq 7.08 \times 10^{-5} \\
4.25 \times 10^{-4} \leq \mathrm{Ek}_{2} \leq 7.08 \times 10^{-5} \\
0.25 \leq \beta \leq 1, \quad \operatorname{Pr}=5.49 \\
0.2 \leq \mathrm{r}_{\mathrm{p}} / \mathrm{r}_{\mathrm{d}} \leq 0.75 \\
227.6 \leq \varepsilon \leq 627.6\end{array}$ \\
\hline $\begin{array}{l}\text { Lallave Cortes } \\
(2009)\end{array}$ & $\begin{array}{l}\text { Partially } \\
\text { Confined }\end{array}$ & Circular & $\begin{aligned} \mathrm{Nu}_{\mathrm{av}}= & 1.94282 \beta^{-0.01} \cdot \mathrm{Re}^{0.74} \cdot \mathrm{Ek}^{-0.1} \cdot\left(\mathrm{b} / \mathrm{d}_{\mathrm{n}}\right)^{-0.05} \\
& .\left(\mathrm{r}_{\mathrm{p}} / \mathrm{r}_{\mathrm{d}}\right)^{-0.05} \cdot \mathrm{Fo}^{-0.01}\end{aligned}$ & $225<\operatorname{Re}<900$ & Numerical & $\begin{array}{l}\text { Spinning Target, } \\
7.08 \times 10^{-5} \leq \mathrm{Ek} \leq 4.25 \times 10^{-4} \\
0.25 \leq \beta \leq 1, \quad \operatorname{Pr}=5.49 \\
0.2 \leq \mathrm{r}_{\mathrm{p}} / \mathrm{r}_{\mathrm{d}} \leq 0.75 \\
0.2 \leq \mathrm{r}_{\mathrm{p}} / \mathrm{r}_{\mathrm{d}} \leq 0.75 \\
0.045 \leq \mathrm{Fo} \leq 0.72 \\
227.6 \leq \varepsilon \leq 376.7\end{array}$ \\
\hline $\begin{array}{l}\text { Fujii and Fujii } \\
\text { (1976) }\end{array}$ & Confined & Circular & $\mathrm{Nu}_{\mathrm{c}, \mathrm{s}}=\left(\frac{\mathrm{Pr}}{4+9 \operatorname{Pr}^{1 / 2}+10 \operatorname{Pr}}\right)^{1 / 5}\left(\operatorname{Gr}_{\mathrm{c}}^{*} \operatorname{Pr}\right)^{1 / 5}$ & Not mentioned & Numerical & - \\
\hline $\begin{array}{l}\mathrm{Li} \text { and } \\
\text { Garimella } \\
(2001)\end{array}$ & $\begin{array}{l}\text { Confined } \\
\text { Submerged }\end{array}$ & Circular & $\begin{aligned} \mathrm{Nu}_{\mathrm{av}}= & 1.064 \operatorname{Re}^{0.513} \operatorname{Pr}^{0.441}\left(\frac{1}{\mathrm{~d}}\right)^{-0.071}\left(\frac{\mathrm{D}_{\mathrm{e}}}{\mathrm{d}}\right)^{-0.266} . \mathrm{A}_{\mathrm{r}} \\
& +1.291 \operatorname{Re}^{0.63} \operatorname{Pr}^{0.441}\left(\frac{\mathrm{D}_{\mathrm{e}}}{\mathrm{d}}\right)^{-1.063} .\left(1-\mathrm{A}_{\mathrm{r}}\right)\end{aligned}$ & $4000<\operatorname{Re}<23000$ & Experimental & $\begin{array}{l}1.59 \mathrm{~mm} \leq \mathrm{d} \leq 6.35 \\
7.1 \leq \operatorname{Pr} \leq 25.2 \\
1 \leq \mathrm{H} / \mathrm{d} \leq 5 \\
0.25 \leq 1 / \mathrm{d} \leq 12 \\
\mathrm{D}_{\mathrm{e}}=11.28 \mathrm{~mm}\end{array}$ \\
\hline $\begin{array}{l}\text { Sung and } \\
\text { Mudawar } \\
(2008)\end{array}$ & Free Surface & Circular & 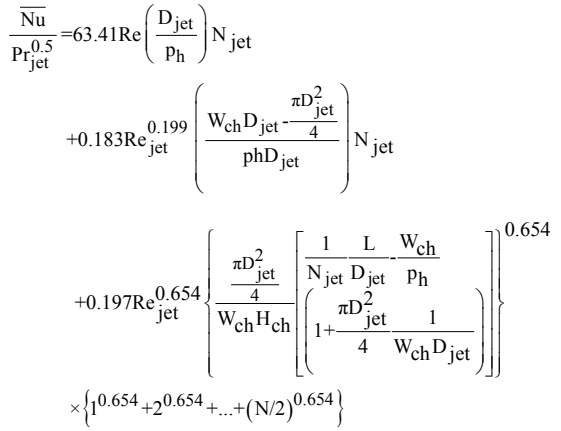 & $1000<\operatorname{Re}<3500$ & $\begin{array}{l}\text { Experimental } \\
\text { and } \\
\text { Numerical }\end{array}$ & $\begin{array}{l}\text { hybrid cooling with N-nozzles: } \\
\text { micro-channel flow and micro- } \\
\text { jet impingement }\end{array}$ \\
\hline
\end{tabular}




\section{LIQUID IMPINGEMENT JETS USING NANOFLUIDS}

In recent years, breakthroughs in manufacturing processes have permitted the creation of solid particles down to the nanometer scale, which in turn has resulted in the birth of a new and rather special class of fluids, called 'nanofluids' (Lee and Choi, 1996, Wang and Mujumdar, 2008a,b). These fluids constitute a very interesting alternative for electronic cooling applications (Keblinski et al., 2005). The term 'nanofluids' usually refers to a mixture composed of a saturated liquid with extremely fine nanoparticles in suspension. Many experimental studies revealed that these innovative working fluids have an extremely high enhancement in thermal conductivity, convective heat transfer coefficient and $\mathrm{CHF}$ in boiling heat transfer (Sung and Mudawar, 2008). It should be mentioned that there is a clear lack of data regarding nanofluid behavior in real thermal applications and some other important issues like the unknown long term effects due to the temperature on the stability and suspension of these special mixtures. In recent years, a few studies on impingement jets using nanofluids have been performed. We believe that a combined system including impingement jets and nanofluids can remove the large amount of heat generated by surfaces in industrial applications such as high performance microelectronic chips.

Microfluid technology is a little older. Microfluids emerged in the beginning of the 1980s, but scientists recognized nanofluids only in 1995. These two new technologies have been used in many industrial researches such as cooling processes but are completely different. Microfluid is the name of a fluid that flows in the micro scale, such as in microchannels, but nanofluids are suspensions of nanoparticles in a base fluid. Microfluids are common in inkjet printheads, DNA chips, fuel cells and microthermal technologies and have some advantages such as small volumes, small size, low energy consumption and effects of the micro domain, while nanofluids are used in heat transfer and lubrication and have the advantage of smaller scale. Nanofluids also relieve minor problems compared to microscale technologies. Such as a lower pressure drop and subsequently lesser pumping power, smaller size and volume, minor erosion and corrosion and more stability.

The use of microfluids in processes involving liquid impingement jets provides greater thermal and flow properties. A new hybrid cooling scheme has been proposed for high-flux thermal management of electronic and power devices. This scheme combines the cooling benefits of micro-channel flow and micro-jet impingement with those of indirect refrigeration cooling. This approach had been used for electronic cooling by Sung and Mudawar (2008). They solved this problem analytically and proposed a new correlation for cases such as, hybrid cooling with N-nozzles.

A few promising works have been performed related to exploiting nanofluids in liquid impingement jets. Roy et al. (2004) were the first to focus on this subject. In the following, we divide important studies into experimental and numerical groups.

\section{EXPERIMENTAL STUDIES}

Nguyen et al. (2008) studied impingement jet heat transfer and erosion effects using nanofluids. They used water- $\mathrm{Al}_{2} \mathrm{O}_{3}$ nanoparticles with $36 \mathrm{~nm}$ average diameter for the cooling of high-power electronic components. The surface heat transfer coefficient increased considerably when the mass flow rate was increased, but was relatively insensitive to the nozzle to heated surface distance. It was found that the use of a nanofluid can provide a heat transfer enhancement of as much as $72 \%$ when compared to water. Results from erosion tests showed that nanofluids have the potential to cause premature wear of mechanical components due to erosion.

Another experimental study was performed by Nguyen et al. (2009) to investigate the heat transfer performance of a $36 \mathrm{~nm} \mathrm{Al}_{2} \mathrm{O}_{3}$-particle-water nanofluids in a confined and submerged impinging jet on a flat, horizontal and circular heated surface. The particle volume fraction ranged from 0 to $6 \%$. Experimental data, obtained for both laminar and turbulent flow regimes, clearly showed that, depending upon the combination of the nozzle to heated surface spacing and particle volume fraction, the use of nanofluids can provide a heat transfer enhancement in some cases; conversely, for other combinations, an adverse effect on the convective heat transfer coefficient may occur. Within the range of experimental parameters used, it was observed that the highest surface heat transfer coefficients were achieved using an intermediate nozzle to surface spacing of 5 $\mathrm{mm}$ and a $2.8 \%$ particle volume fraction. Nanofluids with high particle volume fractions, e.g., $6 \%$ or higher, have been found not to be appropriate for heat transfer enhancement purposes under the confined impinging jet configuration. On the other hand, for a very small and a large nozzle to heated surface spacing, it was observed that nanofluids do 
not provide a perceptible heat transfer enhancement. For some particular cases, they produced a clear decrease of the convective heat transfer coefficient compared to that obtained using distilled water. Such behavior is obviously due to the fact that, with increasing mass flow rate, the forced convection effect also increases considerably, which in turn increases the heat transfer within the fluid.

Li et al. (2011) concentrated on the heat transfer performance of nanofluids in a submerged impingement jet system. Their results demonstrated that nanofluids are promising new coolants compared to pure water. They modified Webb and Ma's (1995) correlation for the nanofluids and proposed a Nusselt predicting correlation as follows:

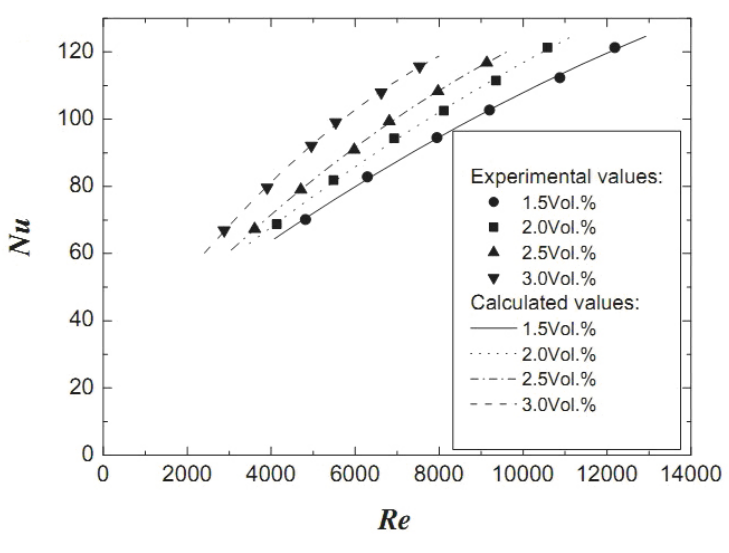

(a)

$$
\begin{aligned}
\mathrm{Nu} & =0.2464\left(1.0+2.2061 \phi^{0.8464} \mathrm{Pe}^{0.2715}\right) \\
& \operatorname{Re}^{0.5375} \operatorname{Pr}^{1 / 3} \\
& \times\left[\begin{array}{l}
1.0+0.3923(\mathrm{H} / \mathrm{D})+0.0086(\mathrm{H} / \mathrm{D})^{2} \\
-0.0259(\mathrm{H} / \mathrm{D})^{3}
\end{array}\right]
\end{aligned}
$$

where, $\mathrm{Nu}, \phi, \mathrm{Pe}, \mathrm{Re}, \mathrm{H}$ and $\mathrm{D}$, are the Nusselt number, volume fraction of nanoparticles, Peclet number, Reynolds number, jet to target distance and diameter of nozzle, respectively. Also, good agreement between results obtained from their experimental study and the prediction model were shown (Figure 4).

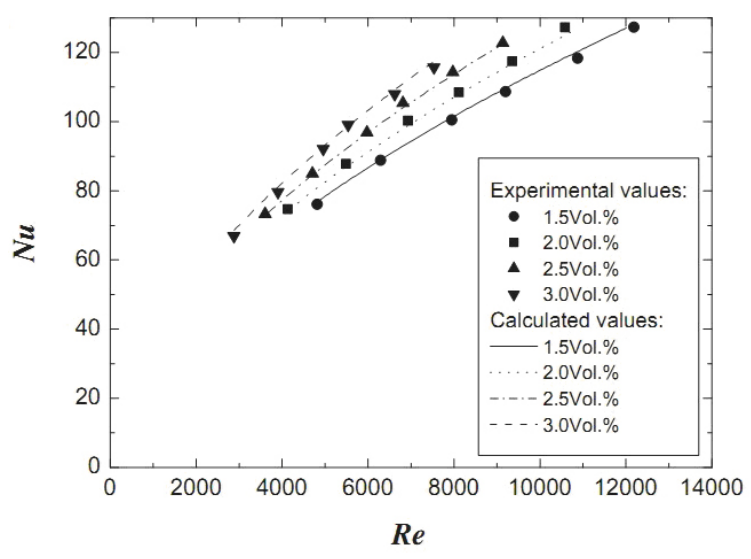

(b)

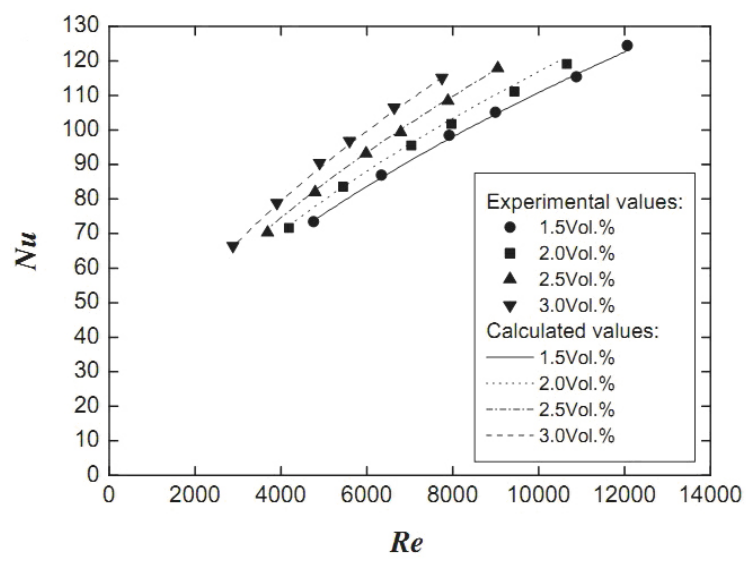

(c)

Figure 4: Comparison between the measured data and the calculated values from correlation (1): $(a) H / D=1$; (b) $\mathrm{H} / \mathrm{D}=2 ;$ (c) $\mathrm{H} / \mathrm{D}=3$ (reprinted from Li et al. (2012), with permission from Elsevier). 
In another study, the jet boiling heat transfer of a water- $\mathrm{CuO}$ particle suspension (nanofluids) jet impingement on a large flat surface was experimentally investigated by Liu and Qiu (2007). The experimental results were compared with those with water. The effects of the nanoparticle concentration and the flow conditions on the nucleate boiling heat transfer and the critical heat flux (CHF) were investigated. The experimental data showed that the jet boiling heat transfer for the water-CuO nanofluids is significantly different from that for water. The surface tension of the nanofluids was equal to about $75 \%$ of that of water, and it hardly changed with different concentrations. The nanofluids have poor nucleate boiling heat transfer compared with the base fluid due to formation of a very thin nanoparticle sorption layer on the heated surface. The reasons were that the solid-liquid contact angle decreased due to a very thin sorption layer on the heated surface and the jet and agitating effect of the nanoparticles in the sub-film layer enhances the supply of liquid to the surface. They concluded that the CHF of nanofluids increased gradually with the increasing particle concentration in the low concentration range, but was constant after the particle concentration exceeded $1 \mathrm{wt} \%$. The CHF for nanofluids increased about $25 \%$ compared with that of water. A sorption layer is formed on the heated surface during jet boiling of nanofluids. After the sorption layer is cleaned out by the water jet, there still exists a very thin coating layer on the surface. The surface roughness and the contact angle are decreased due to the formation of the sorption layer.

\section{NUMERICAL STUDIES}

Roy et al. (2004) performed a numerical study of the heat transfer performance of a liquid impingement jet using $\gamma-\mathrm{Al}_{2} \mathrm{O}_{3}$ nanoparticles suspended in water. They indicated a considerable improvement in heat transfer rates (up to 100\% in local heat transfer coefficient) even at small particle volume fractions.

Most studies related to nanofluids used in impingement jet heat transfer are limited to water- $\mathrm{Al}_{2} \mathrm{O}_{3}$ nanofluids. Almost all works, whether experimental or numerical, utilize a single circular nozzle. Nonetheless, other nanoparticle materials are promising such as carbon nanotubes and $\mathrm{CuO}$. For example, Wang Xiangqi (2007) studied the utilization of this method to cool microelectronic components. He used four types of nanofluids and observed interesting results. For instance, in the case of oil-CNT nanofluids, the results demonstrated a $100 \%$ enhancement in Nusselt number and, therefore, in heat transfer performance.

It should be noted that for electronic chip cooling by nanofluids impingement jets, the use of an array of jets is important and more interesting because of the desired uniform temperature distribution on electronic chip. However, no study using an array of jets involving nanofluids is available.

Another interesting numerical study performed by Palm et al. (2006) used well-known accurate models to predict temperature-dependent nanofluids properties like the effective viscosity and thermal conductivity. Their results demonstrated clearly that the temperature-dependent models gave a higher heat transfer enhancement and lower wall stress compared to the constant properties models.

Heat transfer enhancement and pumping power in an impingement jet using suspended nanoparticles $\left(\mathrm{Al}_{2} \mathrm{O}_{3}\right.$ dispersed in water) was investigated by Gherasim et al. (2011). They demonstrated clearly a direct relation between nanoparticle volume fraction and Nusselt number enhancement. Results indicated that heat transfer enhancement is possible in this application using nanofluids. In general, it was noted that the mean Nusselt number increased with particle volume fraction and Reynolds number and decreased with an increase in disk spacing. On the other hand, the important increase in associated pumping power may impose some limitations on the efficient use of nanofluid in a radial impingement system. Their results are shown in Figures 5 and 6.

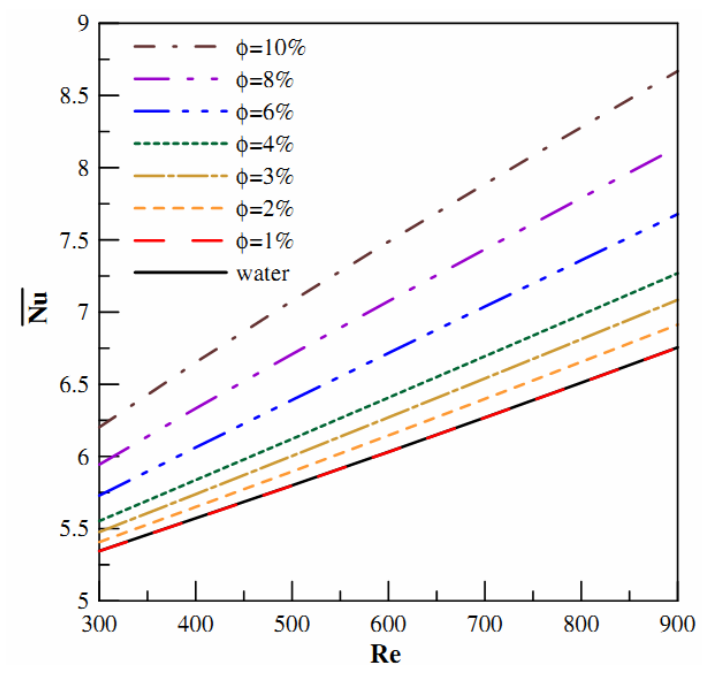

Figure 5: Influence of Reynolds number and particle volume fraction on Nusselt number (reprinted from Yang and Lai (2010), with permission from Elsevier). 


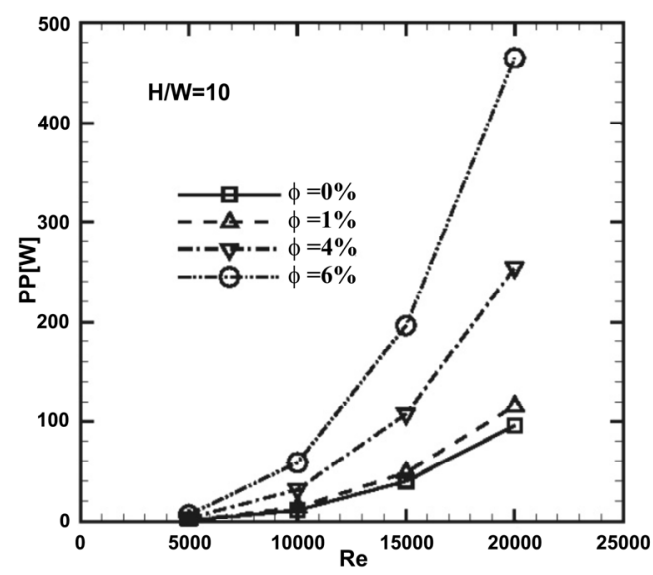

Figure 6: Pumping power required as a function of Reynolds number (Manca et al., 2011).

Manca et al. (2011) studied a confined slot jet with alumina-water nanofluids. The flow is turbulent and a constant temperature is applied on the impinging fluid. Also, a single-phase model approach was adopted. Their local Nusselt number profiles showed the highest values at the stagnation point, and the lowest at the end of the heated plate. The highest values of the average Nusselt numbers increased as the particle concentration and Reynolds numbers increased, the highest values being observed for $\mathrm{H} / \mathrm{W}$ $=10$. A maximum increase of $18 \%$ was detected at a $6 \%$ volume fraction of nanoparticles.

An experimental investigation to study the heat transfer between a vertical round alumina-water nanofluids jet and a horizontal circular round surface was carried out by Zeitoun and Ali (2012). They found that the heating disk diameter shows reverse effect on heat transfer. It was also found that presenting the data in terms of Reynolds number at the impingement jet diameter can take into account both the effects of jet heights and nozzle diameter. Showing the data in terms of Peclet numbers at fixed impingement nozzle diameter makes the data less sensitive to the percentage change of the nanoparticle concentration. Finally, a general heat transfer correlation is obtained verses Peclet numbers using the nanoparticle concentrations and the nozzle diameter ratio:

$$
\begin{aligned}
\mathrm{Nu}= & \left(0.2\left(\frac{\mathrm{D}_{\mathrm{e}}}{\mathrm{D}_{\mathrm{i}}}\right)^{-0.5}-5.022\left(\frac{\mathrm{D}_{\mathrm{e}}}{\mathrm{D}_{\mathrm{i}}}\right)^{-2}\right) \\
& (1-\mathrm{m})^{2.136} \mathrm{Pe}_{\mathrm{i}}^{0.933}
\end{aligned}
$$

To better understand the behavior of liquid impingement jets with nanofluids, we provide two graphs; comparing the results of important studies in this area. It is clear that the heat transfer enhancement increases with any increase in nanoparticle volume fraction and Reynolds number (see Figures 7,8).

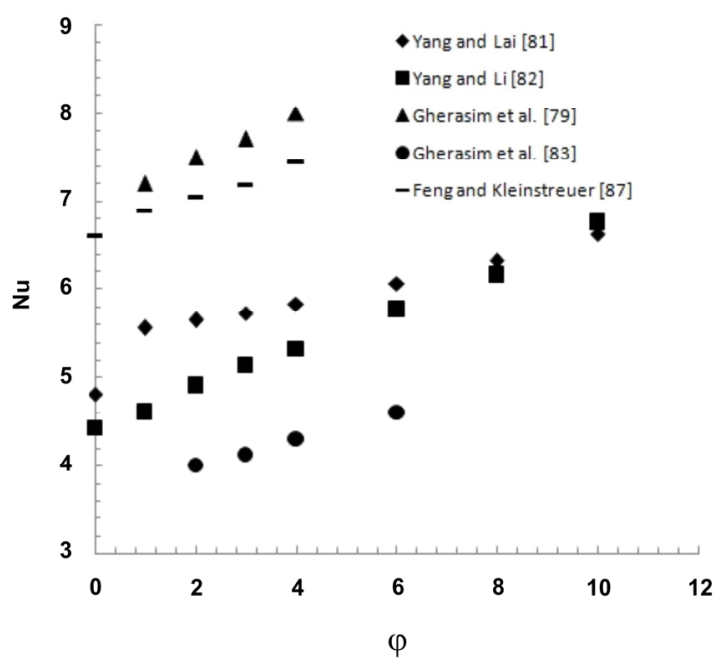

Figure 7: Comparison of some important data for Nusselt number as a function of nanoparticle volume fraction.

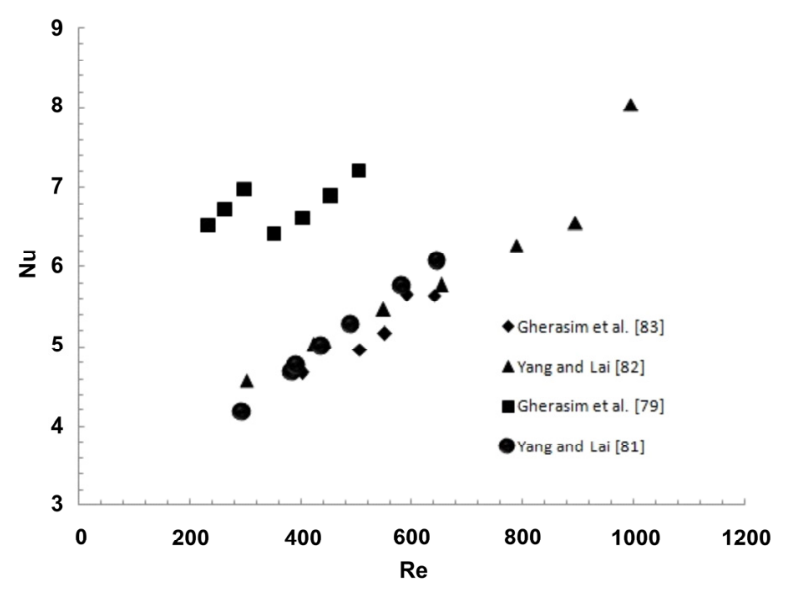

Figure 8: Comparison of some important data for Nusselt number versus Reynolds number.

Table 2 shows more details about liquid impingement jets using Nanofluids such as base fluids, material of the nanoparticles and effective parameters in this area. This table indicates a clear shortage of useful investigations about the use of nanofluids in liquid impingement jets. 
Table 2: Studies done using nanofluids in liquid impingement jet heat transfer

\begin{tabular}{|c|c|c|c|c|c|c|c|c|c|c|c|}
\hline Ref. No. & $\begin{array}{l}\text { Type of } \\
\text { Study }\end{array}$ & $\begin{array}{c}\text { Type of } \\
\text { Jet }\end{array}$ & $\begin{array}{l}\text { Base } \\
\text { Fluid }\end{array}$ & $\begin{array}{c}\text { Nano } \\
\text { particle } \\
\text { Material }\end{array}$ & $\begin{array}{l}\text { Nano } \\
\text { particle } \\
\text { Size }\end{array}$ & $\begin{array}{c}\text { Best } \\
\text { Volume } \\
\text { Fraction }\end{array}$ & $\begin{array}{l}\text { Max. Nusselt } \\
\text { Enhancement }\end{array}$ & $\begin{array}{l}\text { Reynolds } \\
\text { Range }\end{array}$ & $\begin{array}{l}\text { Flow } \\
\text { Regime }\end{array}$ & $\begin{array}{c}\text { Boiling } \\
\text { Phenomena }\end{array}$ & Note \\
\hline $\begin{array}{l}\text { Roy et al. } \\
\text { (2004) }\end{array}$ & Numerical & Confined & Water & $\gamma-\mathrm{Al}_{2} \mathrm{O}_{3}$ & $\begin{array}{c}\text { Not } \\
\text { mentioned }\end{array}$ & $10 \%$ & $110 \%$ & $\begin{array}{l}1200 \text { and } \\
2470\end{array}$ & Laminar & No & Brinkman's formula for viscosity \\
\hline $\begin{array}{l}\text { Nguyen et al. } \\
\text { (2008) }\end{array}$ & Experimental & Submerged & Water & $\mathrm{Al}_{2} \mathrm{O}_{3}$ & $36 \mathrm{~nm}$ & $5 \%$ & $72 \%$ & $1700<\operatorname{Re}<20000$ & Turbulent & No & $\begin{array}{l}\text { Nanofluids have the potential } \\
\text { to cause premature wear of } \\
\text { mechanical components due to } \\
\text { erosion. }\end{array}$ \\
\hline $\begin{array}{l}\text { Nguyen et al. } \\
\quad \text { (2009) }\end{array}$ & Experimental & $\begin{array}{l}\text { Confined } \\
\text { and } \\
\text { Submerged }\end{array}$ & Water & $\mathrm{Al}_{2} \mathrm{O}_{3}$ & $36 \mathrm{~nm}$ & $2.8 \%$ & $\begin{array}{l}\text { Not } \\
\text { mentioned }\end{array}$ & $3800<\operatorname{Re}<88000$ & $\begin{array}{l}\text { Laminar } \\
\text { and } \\
\text { Turbulent }\end{array}$ & No & $\begin{array}{l}\text { High particle volume fractions, } \\
\text { say } 6 \% \text { or higher, have been found } \\
\text { not appropriate for the heat } \\
\text { transfer enhancement purpose } \\
\text { under the confined impinging } \\
\text { jet configuration. }\end{array}$ \\
\hline $\begin{array}{l}\text { Liu and Qiu } \\
\text { (2007) }\end{array}$ & Experimental & $\begin{array}{c}\text { Free } \\
\text { Surface }\end{array}$ & Water & $\mathrm{CuO}$ & $50 \mathrm{~nm}$ & - & Degrading & $25000<\operatorname{Re}<400000$ & Turbulent & Yes & $\begin{array}{l}\text { Surface tension of the nanofluid } \\
\text { was about equal to } 75 \% \text { of that } \\
\text { of water; } \\
\text { The CHF for nanofluid increased } \\
\text { about } 25 \% \text { compared with that } \\
\text { of water. }\end{array}$ \\
\hline $\begin{array}{l}\text { Wang } \\
\text { Xiangqi } \\
(2007)\end{array}$ & Numerical & Confined & $\begin{array}{c}\text { Water/ } \\
\text { water/oil/ } \\
\text { water }\end{array}$ & $\begin{array}{c}\mathrm{Al}_{2} \mathrm{O}_{3} / \\
\text { copper/ } \\
\mathrm{CNT} / \mathrm{CNT} \\
\end{array}$ & - & $\begin{array}{c}10 \% /- \\
/ 1 \% / 10 \%\end{array}$ & $\begin{array}{c}30 \% /- \\
/ 100 \% / 80 \%\end{array}$ & Not mentioned & Laminar & No & $\begin{array}{l}\text { Increases vortex strength as well } \\
\text { as the thermal performance of } \\
\text { the nanofluids. }\end{array}$ \\
\hline $\begin{array}{l}\text { Palm et al. } \\
\quad \text { (2006) }\end{array}$ & Numerical & Confined & Water EG & $\mathrm{Al}_{2} \mathrm{O}_{3}$ & $38 \mathrm{~nm}$ & $7.5 \%$ & $70 \%$ & $500<\operatorname{Re}<946$ & Laminar & No & $\begin{array}{l}\text { Approximately two fold in- } \\
\text { creases in wall shear stress are } \\
\text { found in the case of a water } \\
\mathrm{Al}_{2} \mathrm{O}_{3} \text { nanofluid with a particle } \\
\text { volume fraction of } 5 \% \text {. }\end{array}$ \\
\hline $\begin{array}{l}\text { Gherasim } \\
\text { et al. (2011) }\end{array}$ & Numerical & Confined & Water & $\mathrm{Al}_{2} \mathrm{O}_{3}$ & $47 \mathrm{~nm}$ & $6 \%$ & $\begin{array}{c}\text { Not } \\
\text { mentioned } \\
\text { but } \\
\text { Prominent }\end{array}$ & $100<\operatorname{Re}<750$ & Laminar & No & $\begin{array}{l}\text { Mean Nusselt number was found } \\
\text { to increase with particle volume } \\
\text { fraction, Reynolds number and } \\
\text { a decrease in disk spacing. }\end{array}$ \\
\hline $\begin{array}{l}\text { Oronzio } \\
\text { Manca } \\
(2011)\end{array}$ & Numerical & Confined & Water & $\mathrm{Al}_{2} \mathrm{O}_{3}$ & $38 \mathrm{~nm}$ & $6 \%$. & $18 \%$ & $5000<\operatorname{Re}<20000$ & Turbulent & No & $\begin{array}{l}\text { Slot jet; } \\
\text { The highest values of the } \\
\text { average Nusselt numbers increase } \\
\text { as the particle concentrations } \\
\text { and Reynolds numbers increase } \\
\text { and the highest values are } \\
\text { observed for } \mathrm{H} / \mathrm{W}=10 \text {. }\end{array}$ \\
\hline $\begin{array}{l}\text { Yang and } \\
\text { Lai (2010) }\end{array}$ & Numerical & Confined & Water & $\mathrm{Al}_{2} \mathrm{O}_{3}$ & $47 \mathrm{~nm}$ & $10 \%$ & $20 \%$ & $300<\operatorname{Re}<900$ & Lamniar & No & $\begin{array}{l}\text { Hamilton-Crosser formula for } \\
\text { effective conductivity along } \\
\text { with the equation developed by } \\
\text { Brinkman for effective viscosity } \\
\text { of nanofluid }\end{array}$ \\
\hline $\begin{array}{l}\text { Yang and } \\
\text { Lai (2011) }\end{array}$ & Numerical & Confined & Water & $\mathrm{Al}_{2} \mathrm{O}_{3}$ & $47 \mathrm{~nm}$ & $10 \%$ & $\begin{array}{l}76.6 \% \text { For } \\
\operatorname{Re}=1000\end{array}$ & $300<\operatorname{Re}<1000$ & Laminar & No & $\begin{array}{l}\text { Nusselt number increases with } \\
\text { the increase of Reynolds number } \\
\text { and nanoparticle volume fraction }\end{array}$ \\
\hline $\begin{array}{l}\text { Gherasim } \\
\text { et al. }(2009)\end{array}$ & Experimental & Confined & Water & $\mathrm{Al}_{2} \mathrm{O}_{3}$ & $47 \mathrm{~nm}$ & $6 \%$ & $\begin{array}{l}\text { Not } \\
\text { mentioned } \\
\text { but } \\
\text { Prominent }\end{array}$ & $500<\operatorname{Re}<946$ & Laminar & No & $\begin{array}{l}\text { Nusselt number increases with } \\
\text { particle volume fraction and } \\
\text { Reynolds number and decreases } \\
\text { with an increase in disk spacing. } \\
\text { Brinkman's formula for viscosity }\end{array}$ \\
\hline $\begin{array}{l}\text { Mitra } \text { et al. } \\
\quad \text { (2012) }\end{array}$ & $\begin{array}{c}\text { Experimental } \\
\text { and } \\
\text { Numerical }\end{array}$ & Confined & Water & $\begin{array}{c}\mathrm{TiO} 2, \\
\text { MWCNT }\end{array}$ & $\begin{array}{c}(20-70 \mathrm{~nm}) \\
(20-70 \mathrm{~nm} \\
\text { in diameter } \\
\text { and } 100- \\
500 \mathrm{~nm} \\
\text { in length })\end{array}$ & - & $\begin{array}{l}\text { Not } \\
\text { mentioned } \\
\text { but } \\
\text { Prominent }\end{array}$ & Not mentioned & Laminar & Yes & $\begin{array}{l}\text { The difference in CHF observed } \\
\text { for nanofluids as compared to } \\
\text { water is marginal and doesn't } \\
\text { play a significant role in cooling } \\
\text { rate change observed. }\end{array}$ \\
\hline $\begin{array}{l}\text { Naphon, and } \\
\text { Wongwises } \\
\text { (2011) }\end{array}$ & Experimental & Confined & $\begin{array}{c}\text { Deionized } \\
\text { water }\end{array}$ & $\mathrm{TiO} 2$ & $21 \mathrm{~nm}$ & $0.4 \%$ & $\begin{array}{l}\text { Not } \\
\text { mentioned } \\
\text { but } \\
\text { Prominent }\end{array}$ & Not mentioned & Turbulent & No & $\begin{array}{l}\text { The CPU temperatures obtained } \\
\text { by this cooling system are lower } \\
\text { than those from the conven- } \\
\text { tional liquid cooling system. }\end{array}$ \\
\hline $\begin{array}{l}\text { Di Lorenzo } \\
\text { et al. }(2012)\end{array}$ & Numerical & Confined & Water & $\mathrm{Al}_{2} \mathrm{O}_{3}$ & $30 \mathrm{~nm}$ & $5 \%$ & $32 \%$ & $\operatorname{Re}<500$ & Laminar & No & $\begin{array}{l}\text { Slot Jets, } \\
\text { A maximum increase of } 32 \% \text { in } \\
\text { terms of average heat transfer } \\
\text { coefficients is detected at } \varphi= \\
5 \% \text { for } \mathrm{H} / \mathrm{W}=8 \text {. }\end{array}$ \\
\hline
\end{tabular}

Continuation Table 2 
Continuation Table 2

\begin{tabular}{|c|c|c|c|c|c|c|c|c|c|c|c|}
\hline Ref. No. & $\begin{array}{l}\text { Type of } \\
\text { Study }\end{array}$ & $\begin{array}{c}\text { Type of } \\
\text { Jet }\end{array}$ & $\begin{array}{l}\text { Base } \\
\text { Fluid }\end{array}$ & $\begin{array}{c}\text { Nano } \\
\text { particle } \\
\text { Material }\end{array}$ & $\begin{array}{l}\text { Nano } \\
\text { particle } \\
\text { Size }\end{array}$ & $\begin{array}{c}\text { Best } \\
\text { Volume } \\
\text { Fraction }\end{array}$ & $\begin{array}{l}\text { Max. Nusselt } \\
\text { Enhancement }\end{array}$ & $\begin{array}{c}\text { Reynolds } \\
\text { Range }\end{array}$ & $\begin{array}{c}\text { Flow } \\
\text { Regime }\end{array}$ & \begin{tabular}{|c|} 
Boiling \\
Phenomena
\end{tabular} & Note \\
\hline $\begin{array}{l}\text { Feng } \\
(2010)\end{array}$ & Numerical & Confined & Water & $\mathrm{Al}_{2} \mathrm{O}_{3}$ & $30-47 \mathrm{~nm}$ & $4 \%$ & $\begin{array}{l}\text { Not } \\
\text { mentioned } \\
\text { but } \\
\text { Prominent }\end{array}$ & $\operatorname{Re}<800$ & Laminar & No & $\begin{array}{l}\text { The results indicate that nano- } \\
\text { fluids are when compared to } \\
\text { pure water. Specifically, smoother } \\
\text { mixture flow fields and tem- } \\
\text { perature distributions can be } \\
\text { achieved. }\end{array}$ \\
\hline $\begin{array}{c}\text { Zeitoun and } \\
\text { Ali (2012) }\end{array}$ & Experimental & $\begin{array}{l}\text { Free } \\
\text { Surface }\end{array}$ & Water & $\mathrm{Al}_{2} \mathrm{O}_{3}$ & $10 \mathrm{~nm}$ & $10 \%$ & $75 \%$ & $2500<\operatorname{Re}<24000$ & Turbulent & No & $\begin{array}{l}\text { General heat transfer correlation } \\
\text { is obtained verses Peclet numbers } \\
\text { using nanoparticle concentra- } \\
\text { tions and the nozzle diameter } \\
\text { ratio as parameters. }\end{array}$ \\
\hline $\begin{array}{l}\text { Li et al. } \\
\text { (2012) }\end{array}$ & Experimental & Submerged & Water & $\mathrm{Cu}$ & $\begin{array}{c}25,100 \\
\mathrm{~nm}\end{array}$ & $3 \%$ & $52 \%$ & $3000<\operatorname{Re}<16000$ & Turbulent & No & $\begin{array}{l}\text { By considering the effects of the } \\
\text { suspended nanoparticles as well } \\
\text { as the condition of impinging } \\
\text { jet, a new heat transfer correla- } \\
\text { tion of nanofluids for the sub- } \\
\text { merged single jet impingement } \\
\text { has been proposed. }\end{array}$ \\
\hline $\begin{array}{l}\text { Roy et al. } \\
(2004)\end{array}$ & Numerical & Confined & Water & $\gamma-\mathrm{Al}_{2} \mathrm{O}_{3}$ & \begin{tabular}{|c|} 
Not \\
mentioned
\end{tabular} & $10 \%$ & $110 \%$ & $\begin{array}{c}1200 \text { and } \\
2470\end{array}$ & Laminar & No & Brinkman's formula for viscosity \\
\hline $\begin{array}{l}\text { Nguyen et al. } \\
\text { (2008) }\end{array}$ & Experimental & Submerged & Water & $\mathrm{Al}_{2} \mathrm{O}_{3}$ & $36 \mathrm{~nm}$ & $5 \%$ & $72 \%$ & $1700<\operatorname{Re}<20000$ & Turbulent & No & $\begin{array}{l}\text { Nanofluids have the potential to } \\
\text { cause premature wear of } \\
\text { mechanical components due to } \\
\text { erosion. }\end{array}$ \\
\hline $\begin{array}{l}\text { Nguyen et al. } \\
\text { (2009) }\end{array}$ & Experimental & $\begin{array}{c}\text { Confined } \\
\text { and } \\
\text { Submerged }\end{array}$ & Water & $\mathrm{Al}_{2} \mathrm{O}_{3}$ & $36 \mathrm{~nm}$ & $2.8 \%$ & $\begin{array}{c}\text { Not } \\
\text { mentioned }\end{array}$ & $3800<\operatorname{Re}<88000$ & $\begin{array}{l}\text { Laminar } \\
\text { and } \\
\text { Turbulent }\end{array}$ & No & $\begin{array}{l}\text { High particle volume fractions, } \\
\text { say } 6 \% \text { or higher, have been } \\
\text { found not appropriate for the } \\
\text { heat transfer enhancement purpose } \\
\text { under the confined impinging je } \\
\text { configuration. }\end{array}$ \\
\hline $\begin{array}{l}\text { Li et al. } \\
\text { (2011) }\end{array}$ & Experimental & Submerged & Water & $\mathrm{Cu}$ & $25-100 \mathrm{~nm}$ & $3 \%$ & $52 \%$ & $2000<\operatorname{Re}<16000$ & Turbulent & No & $\begin{array}{l}\text { A new heat transfer correlation } \\
\text { of nanofluids for the submerged } \\
\text { single jet impingement has been } \\
\text { proposed. }\end{array}$ \\
\hline $\begin{array}{l}\text { Liu and Qiu } \\
\text { (2007) }\end{array}$ & Experimental & $\begin{array}{l}\text { Free } \\
\text { Surface }\end{array}$ & Water & $\mathrm{CuO}$ & $50 \mathrm{~nm}$ & - & Degrading & $25000<\operatorname{Re}<400000$ & Turbulent & Yes & $\begin{array}{l}\text { Surface tension of the nanofluid } \\
\text { was about equal to } 75 \% \text { of that } \\
\text { of water; } \\
\text { The CHF for nanofluid increased } \\
\text { about } 25 \% \text { compared with that } \\
\text { of water. }\end{array}$ \\
\hline $\begin{array}{l}\text { Wang } \\
\text { Xiangqi } \\
(2007)\end{array}$ & Numerical & Confined & $\begin{array}{c}\text { Water/ } \\
\text { water/oil/ } \\
\text { water }\end{array}$ & \begin{tabular}{|c|}
$\mathrm{Al}_{2} \mathrm{O}_{3} /$ \\
copper/ \\
$\mathrm{CNT} / \mathrm{CNT}$
\end{tabular} & - & $\begin{array}{c}10 \% /- \\
/ 1 \% / 10 \%\end{array}$ & $\begin{array}{c}30 \% /- \\
/ 100 \% / 80 \%\end{array}$ & Not mentioned & Laminar & No & $\begin{array}{l}\text { Increases vortex strength as } \\
\text { well as the thermal performance } \\
\text { of the nanofluids. }\end{array}$ \\
\hline $\begin{array}{l}\text { Palm et al. } \\
\quad \text { (2006) }\end{array}$ & Numerical & Confined & $\begin{array}{c}\text { Water } \\
\text { EG }\end{array}$ & $\mathrm{Al}_{2} \mathrm{O}_{3}$ & $38 \mathrm{~nm}$ & $7.5 \%$ & $70 \%$ & $500<\operatorname{Re}<946$ & Laminar & No & $\begin{array}{l}\text { Approximately two fold in- } \\
\text { creases in wall shear stress are } \\
\text { found in the case of a water } \\
\mathrm{Al}_{2} \mathrm{O}_{3} \text { nanofluid with a particle } \\
\text { volume fraction of } 5 \% \text {. }\end{array}$ \\
\hline $\begin{array}{l}\text { Gherasim } \\
\text { et al. }(2011)\end{array}$ & Numerical & Confined & Water & $\mathrm{Al}_{2} \mathrm{O}_{3}$ & $47 \mathrm{~nm}$ & $6 \%$ & $\begin{array}{l}\text { Not } \\
\text { mentioned } \\
\text { but } \\
\text { Prominent }\end{array}$ & $100<\operatorname{Re}<750$ & Laminar & No & $\begin{array}{l}\text { Mean Nusselt number was found } \\
\text { to increase with particle volume } \\
\text { fraction, Reynolds number and } \\
\text { a decrease in disk spacing. }\end{array}$ \\
\hline $\begin{array}{l}\text { Oronzio } \\
\text { Manca } \\
(2011)\end{array}$ & Numerical & Confined & Water & $\mathrm{Al}_{2} \mathrm{O}_{3}$ & $38 \mathrm{~nm}$ & $6 \%$. & $18 \%$ & $5000<\operatorname{Re}<20000$ & Turbulent & No & $\begin{array}{l}\text { Slot jet; } \\
\text { The highest values of the aver- } \\
\text { age Nusselt numbers increase as } \\
\text { the particle concentrations and } \\
\text { Reynolds numbers increase and } \\
\text { the highest values are observed } \\
\text { for } \mathrm{H} / \mathrm{W}=10 \text {. }\end{array}$ \\
\hline $\begin{array}{l}\text { Yang and } \\
\text { Lai (2010) }\end{array}$ & Numerical & Confined & Water & $\mathrm{Al}_{2} \mathrm{O}_{3}$ & $47 \mathrm{~nm}$ & $10 \%$ & $20 \%$ & $300<\operatorname{Re}<900$ & Lamniar & No & $\begin{array}{l}\text { Hamilton-Crosser formula for } \\
\text { effective conductivity along with } \\
\text { the equation developed by } \\
\text { Brinkman for effective viscosity } \\
\text { of nanofluid }\end{array}$ \\
\hline $\begin{array}{l}\text { Yang and } \\
\text { Lai (2011) }\end{array}$ & Numerical & Confined & Water & $\mathrm{Al}_{2} \mathrm{O}_{3}$ & $47 \mathrm{~nm}$ & $10 \%$ & $\begin{array}{l}76.6 \% \text { for } \\
\operatorname{Re}=1000\end{array}$ & $300<\operatorname{Re}<1000$ & Laminar & No & $\begin{array}{l}\text { Nusselt number increases with } \\
\text { the increase of Reynolds number } \\
\text { and nanoparticle volume fraction }\end{array}$ \\
\hline
\end{tabular}

Continuation Table 2 
Continuation Table 2

\begin{tabular}{|c|c|c|c|c|c|c|c|c|c|c|c|}
\hline Ref. No. & $\begin{array}{l}\text { Type of } \\
\text { Study }\end{array}$ & $\begin{array}{c}\text { Type of } \\
\text { Jet }\end{array}$ & $\begin{array}{l}\text { Base } \\
\text { Fluid }\end{array}$ & $\begin{array}{c}\text { Nano } \\
\text { particle } \\
\text { Material }\end{array}$ & $\begin{array}{c}\text { Nano } \\
\text { particle } \\
\text { Size }\end{array}$ & $\begin{array}{c}\text { Best } \\
\text { Volume } \\
\text { Fraction }\end{array}$ & $\begin{array}{l}\text { Max. Nusselt } \\
\text { Enhancement }\end{array}$ & $\begin{array}{c}\text { Reynolds } \\
\text { Range }\end{array}$ & $\begin{array}{c}\text { Flow } \\
\text { Regime }\end{array}$ & $\begin{array}{c}\text { Boiling } \\
\text { Phenomena }\end{array}$ & Note \\
\hline $\begin{array}{c}\text { Gherasim } \\
\text { et al. (2009) }\end{array}$ & Experimental & Confined & Water & $\mathrm{Al}_{2} \mathrm{O}_{3}$ & $47 \mathrm{~nm}$ & $6 \%$ & $\begin{array}{l}\text { Not } \\
\text { mentioned } \\
\text { but } \\
\text { Prominent }\end{array}$ & $500<\operatorname{Re}<946$ & Laminar & No & $\begin{array}{l}\text { Nusselt number increases with } \\
\text { particle volume fraction and } \\
\text { Reynolds number and decreases } \\
\text { with an increase in disk spacing; } \\
\text { Brinkman's formula for viscosity }\end{array}$ \\
\hline $\begin{array}{l}\text { Mitra et al. } \\
\quad \text { (2012) }\end{array}$ & $\begin{array}{c}\text { Experimental } \\
\text { and } \\
\text { Numerical }\end{array}$ & Confined & Water & $\begin{array}{c}\text { TiO2, } \\
\text { MWCNT }\end{array}$ & $\begin{array}{c}(20-70 \mathrm{~nm}), \\
(20-70 \mathrm{~nm} \\
\text { in diameter } \\
\text { and } 100- \\
500 \mathrm{~nm} \\
\text { in length) }\end{array}$ & - & $\begin{array}{l}\text { Not } \\
\text { mentioned } \\
\text { but } \\
\text { Prominent }\end{array}$ & Not mentioned & Laminar & Yes & $\begin{array}{l}\text { The difference in CHF observed } \\
\text { for nanofluids as compared to } \\
\text { water is marginal and doesn't } \\
\text { play a significant role in cooling } \\
\text { rate change observed. }\end{array}$ \\
\hline $\begin{array}{c}\text { Naphon, and } \\
\text { Wongwises } \\
(2011)\end{array}$ & Experimental & Confined & $\begin{array}{l}\text { Deionized } \\
\text { water }\end{array}$ & $\mathrm{TiO} 2$ & $21 \mathrm{~nm}$ & $0.4 \%$ & $\begin{array}{l}\text { Not } \\
\text { mentioned } \\
\text { but } \\
\text { Prominent }\end{array}$ & Not mentioned & Turbulent & No & $\begin{array}{l}\text { The CPU temperatures obtained } \\
\text { by this cooling system are lower } \\
\text { than those from the conven- } \\
\text { tional liquid cooling system. }\end{array}$ \\
\hline $\begin{array}{l}\text { Di Lorenzo } \\
\text { et al. }(2012)\end{array}$ & Numerical & Confined & Water & $\mathrm{Al}_{2} \mathrm{O}_{3}$ & $30 \mathrm{~nm}$ & $5 \%$ & $32 \%$ & $\operatorname{Re}<500$ & Laminar & No & $\begin{array}{l}\text { Slot Jets, } \\
\text { A maximum increase of } 32 \% \text { in } \\
\text { terms of average heat transfer } \\
\text { coefficients is detected at } \varphi= \\
5 \% \text { for } \mathrm{H} / \mathrm{W}=8 \text {. }\end{array}$ \\
\hline $\begin{array}{l}\text { Feng } \\
(2010)\end{array}$ & Numerical & Confined & Water & $\mathrm{Al}_{2} \mathrm{O}_{3}$ & $30-47 \mathrm{~nm}$ & $4 \%$ & $\begin{array}{l}\text { Not } \\
\text { mentioned } \\
\text { but } \\
\text { Prominent }\end{array}$ & $\operatorname{Re}<800$ & Laminar & No & $\begin{array}{l}\text { The results indicate that nano- } \\
\text { fluids are when compared to } \\
\text { pure water. Specifically, smoother } \\
\text { mixture flow fields and tem- } \\
\text { perature distributions can be } \\
\text { achieved. }\end{array}$ \\
\hline
\end{tabular}

\section{CONCLUSION}

In this study, we concentrated on a liquid impingement jet and its combination with nanofluids. Thorough reviewing important studies, we concluded that,

- The Nusselt number grows as the Reynolds number increases, whether in the cases involving nanofluids or pure fluids.

- The Nusselt number enhances with the particle volume fraction and decreases with an increase in the nozzle to target distance.

- The increase in pressure drop is significant with the presence of nanoparticles.

The parameters that affected the Nusselt number in liquid impingement jets are fluid properties, the geometry of jets, jet velocity, Reynolds number, Prandtl number, nozzle to target distance, material properties of the target, porosity of the target, jet subcooling for boiling phenomena and nozzle diameter. It seems that there is a need for more study of physical properties and the porosity of target and jet to jet interaction before and after impinging. Different arrays of multiple jets should also be investigated for an acceptable optimization between desirability of more jets and the problem of enhancing pressure drop. The few studies on impingement jets with nanofluids that have been performed and their interesting aspects are shown. However, for the acceptance of these promising characteristics the heat transfer community needs more evidences. More studies are required to develop models for Nusselt number prediction using nanofluids. Also, there is no study of an array of liquid impingement jets using nanofluids and such a study should be performed in the near future to determine the effects of nanoparticles on the heat transfer performance of multiple jets systems.

\section{NOMENCLATURE}

$\mathrm{A}_{\text {corr }} \quad$ Area corresponding to a $\mathrm{m}^{2}$ single jet

$\mathrm{A}_{\mathrm{r}} \quad$ Ratio of impingement region area to target area, $(1.9 \mathrm{~d})^{2} / \mathrm{D}_{\mathrm{e}}^{2}$

B Slot jet nozzle width m

$\mathrm{d}_{\mathrm{i}}, \mathrm{D}$ Diameter of nozzle m

$\mathrm{D}_{\mathrm{e}} \quad$ Effective target diameter $\quad\left(4 \mathrm{~A}_{\mathrm{h}} / \pi\right)^{1 / 2}$

$\mathrm{D}_{\mathrm{i}} \quad$ Jet diameter at impingement $\quad \mathrm{m}$

$\mathrm{d}_{\mathrm{n}}$ Jet diameter m

Ek Ekman number, $\mathrm{Ek}=v_{\mathrm{f}} /\left(4 \Omega \mathrm{r}_{\mathrm{d}}^{2}\right)$

$\mathrm{Ek}_{1} \quad$ Ekman number, $\mathrm{Ek}_{1}=v_{\mathrm{f}} /\left(4 \Omega_{1} \mathrm{r}_{\mathrm{d}}^{2}\right)$ 
$\mathrm{Ek}_{2} \quad$ Ekman number,

$$
\mathrm{Ek}_{2}=v_{\mathrm{f}} /\left(4 \Omega_{2} \mathrm{r}_{\mathrm{d}}^{2}\right)
$$

$\mathrm{Gr}_{\mathrm{c}}^{*} \quad$ Modified Grashof number at the center of the heater

$\mathrm{H}, \mathrm{L} / \mathrm{D}$ Jet-to-target distance

k Thermal conductivity

n Number of jets.

L Half of the heat source length, average length of the wall jet region

$\mathrm{L}_{\mathrm{e}} \quad$ Length of the nozzle unit cell for an array

L/D Nozzle-to-surface distance

1 Length of the side of the square heat source, orifice thickness

m Nanofluid particle mass concentration

$\mathrm{Nu} \quad$ Nusselt number

$\mathrm{Nu}_{0} \quad$ Nusselt number at the stagnation point

$\mathrm{Nu}_{\mathrm{c}, \mathrm{s}} \quad$ Nusselt number at the center of the heater

Pr Prandtl number

$\mathrm{Pe} \quad$ Peclet number

$\mathrm{Pe} \quad$ Peclet number at impingement

$\mathrm{R}$ Equivalent radius of the target

Re Reynolds number

$\mathrm{r} \quad$ Radial distance from the stagnation point

$\mathrm{r}_{\mathrm{p}} / \mathrm{r}_{\mathrm{d}} \quad$ Confinement plate to disk radius ratio (confinement ratio)

S Nozzle pitch (distance between neighboring nozzles in a square array)

$\mathrm{S}_{\mathrm{NP}} \quad$ Nozzle-to-surface distance.

$\mathrm{s} \quad$ Jet-to-jet spacing

$\mathrm{T} \quad$ Temperature

$\mathrm{T}_{\mathrm{s}} \quad$ Surface temperature

W Nozzle width

$\mathrm{X}, \mathrm{Y}$ Non-dimensional Cartesian coordinates,

$\mathrm{X}=\mathrm{x} / \mathrm{L}, \quad \mathrm{Y}=\mathrm{y} / \mathrm{L}$

$\mathrm{z} \quad$ Nozzle exit to impingement plate spacing

$\mathrm{p}_{\mathrm{h}} \quad$ Perimeter of micro-channel, $2\left(\mathrm{~W}_{\mathrm{ch}}+\mathrm{H}_{\mathrm{ch}}\right)$

\section{Greek Symbols}

$\varepsilon \quad$ Thermal conductivity ratio, $\varepsilon=\mathrm{k}_{\mathrm{s}} / \mathrm{k}_{\mathrm{f}}$, $\alpha_{\mathrm{J}}=\left(\pi \mathrm{d}^{2}\right) /\left(4 \mathrm{~A}_{\text {corr }}\right)$

$\mathrm{W} / \mathrm{m} . \mathrm{K} \quad \theta_{\mathrm{f}} \quad$ Non-dimensional fluid temperature,

$\mathrm{m}$ $\theta_{\mathrm{f}}=\left(\mathrm{T}_{\mathrm{f}}-\mathrm{T}_{\mathrm{c}}\right) /\left(\mathrm{T}_{\mathrm{h}}-\mathrm{T}_{\mathrm{c}}\right)$

$\theta_{\mathrm{s}} \quad$ Non-dimensional solid temperature,

$\mathrm{m}$ $\theta_{\mathrm{s}}=\left(\mathrm{T}_{\mathrm{s}}-\mathrm{T}_{\mathrm{c}}\right) /\left(\mathrm{T}_{\mathrm{h}}-\mathrm{T}_{\mathrm{c}}\right)$

$\phi \quad$ Volume fraction of nanoparticles

$\theta \quad$ Jet angle.

degree

$\mathrm{m}$

$\beta$

Dimensionless nozzle-toplate spacing, $\mathrm{H}_{\mathrm{n}} / \mathrm{d}_{\mathrm{n}}$

\section{Subscript}

n nozzle

s boundary layer solution,

solid phase

$\mathrm{t}$ total (fluid + solid)

c cold wall

h hot wall

f fluid phase

i inlet

$\mathrm{m}$

o outlet

$\mathrm{m}$

\section{REFERENCES}

Aihara, T. and Kim, J. K., Boiling heat transfer of a microimpinging jet of liquid nitrogen in a very slender cryoprobe. International Journal of Heat and Mass Transfer, vol. 1, no. 36, pp. 169-175 (1993).

Amano, R. S. and Brandt, H., Numerical study of turbulent axisymmetric jets impinging on a plate and flowing into an axisymmetric cavity. Journal of Fluid Engineering, vol. 106, pp. 410-417 (1984).

Ashforth-Frost, S. and Rudel, U. W., Thermal and hydrodynamic visualisation of a water jet impinging on a flat surface using microencapsulated liquid crystals. International Journal of Fluid Dynamics, vol. 7, pp. 1-7 (2002).

Banooni, S., Hosseinalipour, S. M., Mujumdar, A. S., Taheran, E. and Mashaiekhi, M., Impingement heat transfer effects on baking of flat bread, drying technology. Drying Technology, vol. 26, pp. 910-919 (2008). 
Bhunia, S. K., Impingement splattering and surface disturbances evolution on turbulent liquid jets in gases. Ph.D. Thesis on Mechanical Engineering, Massachusetts Institute of Technology, United States (1993).

Bunker, R. S., Gas turbine heat transfer: ten remaining hot gas path challenges. Journal of Turbomachinery, vol. 129, pp. 193-201 (2007).

Carper Jr., H. J. and Deffenbaugh, D. M., Heat transfer from a rotating disk with liquid jet impingement. In: Proceedings of the Sixth International Heat Transfer Conference, Toronto, Canada, vol. 4, Hemisphere Public Corporation, Washington, DC, pp. 113-118 (1978).

Chen, Y. C., Ma, C. F., Qin, M. and Li, X., Theoretical study on impingement heat transfer with singlephase free-surface slot jets. International Journal of Heat and Mass Transfer, vol. 48, pp. 33813386 (2005).

Choi, S. U. S., Enhancement thermal conductivity of fluids with nanoparticles. Developments and applications of non-Newtonian flows. In: Siginer, D. A. and Wang, H. P. (Eds.), ASME Publications, FED 231/MD, 66, pp. 99-105 (1995).

Chung, Y. M., Luo, K. H. and Sandham, N. D., Numerical study of momentum and heat transfer in unsteady impinging jets. International Journal of Heat and Fluid Flow, vol. 23, pp. 592-600 (2002).

Churchill, S. W. and Bernstein, M., A correlating equation for forced convection from gases and liquids to a circular cylinder in crossflow. ASME Journal of Heat and Transfer, vol. 99, pp. 300306 (1977).

Cooper, D., Jackson, D. C., Launder, B. E. and Liao, G. X., Impinging jet studies for turbulence model assessment: Flow-field experiments. International Journal of Heat and Mass Transfer, vol. 36, pp. 2675-2684 (1993).

Di Lorenzo, G., Manca, O., Nardini, S. and Ricci, D., Numerical study of laminar confined impinging slot jets with nanofluids. Advances in Mechanical Engineering, Article ID 248795, DOI:10.1155/2012/ 248795 (2012).

Fang, L. and Kasagi, N., Heat transfer characteristics of a micro-scale axisymmetric liquid impinging jet. $44^{\text {th }}$ Japan Heat Transfer Symposium, vol. 44 (2007).

Feng, Y. and Kleinstreuer, C., Nanofluid convective heat transfer in a parallel-disk system. International Journal of Heat and Mass Transfer, vol. 53, pp. 4619-4628 (2010).

Fitzgerald, J. A. and Garimella, S. V., A study of the flow field of a confined and submerged impinging jet. International Journal of Heat and Mass Transfer, vol. 41, pp. 1025-1034 (1998).

Fujii, T. and Fujii, M., The dependence of local Nusselt number on Prandtl number in the case of free convection along a vertical surface with uniform heat flux. International Journal of Heat and Mass Transfer, vol. 19, no. 10, pp. 121-122 (1976).

Garimella, S. V. and Rice, R. A., Confined and submerged liquid jet impingement jet heat transfer. ASME Journal of Heat Transfer, vol. 117, pp. 871-877 (1995).

Gherasim, I., Roy, G., Nguyen, C. T. and Vo-Ngoc, D., Experimental investigation of nanofluids in confined laminar radial flows. International Journal of Thermal Sciences, vol. 48, pp. 14861493 (2009).

Gherasim, I., Roy, G., Nguyen, C. T. and Vo-Ngoc, D., Heat transfer enhancement and pumping power in confined radial flows using nanoparticle suspensions (nanofluids). International Journal of Thermal Sciences, vol. 50, pp. 369-377 (2011).

Goldstein, R. J. and Timmers, J. F., Visualization of heat transfer from arrays of impinging jets. International Journal of Heat and Mass Transfer, vol. 25, pp. 1857-1868 (1982).

Jiji, L. M. and Dagan, Z., Experimental investigation of single phase multi-jet impingement cooling of an array of microelectronic heat sources. In: Proceedings of the International Symposium of Cooling Technology for Electronic Equipment, The Pacific Institute for Thermal Engineering, HI, pp. 265 (1987).

Kandlikar, S. G., Heat transfer characteristics in partial boiling, fully developed boiling, and significant void flow regions of subcooled flow boiling. ASME Journal of Heat Transfer, vol. 5, no. 120, pp. 395-402 (1998).

Keblinski, P., Eastman, J. A. and Cahill, D. G., Nanofluids for thermal transport. Materials Today, pp. 36-44 (2005).

Kiryo, M., Development of oil-cooled 750 cc motorcycle engine. Automobile Technology, vol. 40, pp. 1154-1158 (1986). (In Japanese).

Kohing, F. C., Waterfall: Water cooling systems. Iron Steel Engineering, vol. 6, no. 62, pp. 30-36 (1985).

Lallave, J. C., Rahman, M. M. and Kumar, A., Numerical analysis of heat transfer on a rotating disk surface under confined liquid jet impingement. International Journal of Heat and Fluid Flow, vol. 28, pp. 720-734 (2007).

Lallave-Cortes, J. C., Numerical heat transfer during partially-confined, confined, and free liquid jet 
impingement with rotation and chemical mechanical planarization process modeling. PhD Thesis in Mechanical Engineering, University of South Florida, USA (2009).

Lee, S. and Choi, S. U. S., Application of metallic nanoparticle suspensions in advanced cooling systems. ASME Publications, PVP 342/MD 72, pp. 227-234 (1996).

Li, C. V. and Garimella, S. V., Prandtl-number effects and generalized correlation for confined and submerged jet impingement. International Journal of Heat and Mass Transfer, vol. 4, pp. 3471-3480 (2001).

Li, Q., Xuan, Y. and Yu, F., Experimental investigation of submerged single jet impingement using $\mathrm{Cu}$ water nanofluid. Applied Thermal Engineering, vol. 36, pp. 426-433 (2012).

Leinhard, V. J. H., Liu, X. and Gabour, L. A., Splattering and heat transfer during impingement of a turbulent liquid jet. ASME Journal of Heat Transfer, 114, (2), pp. 362-372 (1992).

Liu X., Leinhard, V. J. H. and Lombara, J. S., Convective heat transfer by impingement of circular liquid jets. ASME Journal of Heat Transfer, vol. 113, pp. 517-582 (1991).

Liu, X., Gabour, L. A., Stagnation point heat transfer during impingement of laminar liquid jets: Analysis including surface tension. ASME Journal of Heat Transfer, vol. 115, no.8, pp. 99-105 (1993).

Liu, Z., Qiu, Y., Boiling heat transfer characteristics of nanofluids jet impingement on a plate surface. International Journal of Heat and Mass Transfer, vol. 43, pp. 699-706 (2007).

Lupton, T. L., Murray, D. B. and Robinson, A. J., The effect of varying confinement levels on the heat transfer to a miniature impinging air jet. Eurotherm, Eindhoven, Netherlands (2008).

Maddox, D. E. and Bar-Cohen, A., Single-phase thermofluid design of submerged-jet impingement cooling for electronic components. ASME Journal of Electronic Packaging, vol. 116, pp. 237 (1994).

Manca, O., Mesolella, P., Nardini, S. and Ricci, D., Numerical study of a confined slot impinging jet with nanofluids. Nanoscale Research Letters, 6:188, pp. 1-16 (2011).

Martin, H., Heat and mass transfer between impinging gas jets and solid surfaces. Advances in Heat Transfer, vol. 13, pp. 1-60 (1977).

Miller, R. S., Madnia, C. K. and Givi, P., Numerical simulation of non-circular jets. Computers and Fluids, vol. 24, pp. 1-25 (1995).

Mitra, S., Saha, S. K., Chakraborty S. and Das, S., Study on boiling heat transfer of water- $\mathrm{TiO}_{2}$ and water-MWCNT nanofluids based laminar jet im- pingement on heated steel surface. Applied Thermal Engineering, vol. 37, pp. 353-359 (2012).

Mudawar, I. and Wadsworth, D. C., Critical heat flux from a simulated chip to a confine rectangular impinging jet of dielectric liquid. International Journal of Heat and Mass Transfer, vol. 5, no. 34, pp. 1465-1479 (1991).

Naphon, P. and Wongwises, S., Experimental study of jet nanofluids impingement system for cooling computer processing unit. Journal of Electronics Cooling and Thermal Control, vol. 1, pp. 1-7 (2011).

Nguyen, C. T., Laplante, G., Cury, M and Simon, G., Experimental investigation of impinging jet heat transfer and erosion effect using $\mathrm{Al}_{2} \mathrm{O}_{3}$-water nanofluid. 6th IASME/WSEAS International Conference on Fluid Mechanics and Aerodynamics (FMA'08), pp. 44-49, Rhodes, Greece (2008).

Nguyen, C. T., Galanis, N., Polidori, G., Fohanno, S., Popa, C. V. and Le Bechec, A., An experimental study of a confined and submerged impinging jet heat transfer using $\mathrm{Al}_{2} \mathrm{O}_{3}$-water nanofluid. International Journal of Thermal Sciences, vol. 48, pp. 401-411 (2009).

Oliphant, K., Webb, B. W. and McQuay, M. Q., An experimental comparison of liquid jet array and spray impingement cooling in the non-boiling regime. Experimental Thermal and Fluid Science, vol. 18, pp. 1-10 (1998).

Palm, S. J., Roy, G. and Nguyen, C. T., Heat transfer enhancement with the use of nanofluids in radial flow cooling systems considering temperaturedependent properties. Applied Thermal Engineering, vol. 26, pp. 2209-2218 (2006).

Pan, Y. and Webb, B. W., Heat transfer characteristics of arrays of free-surface liquid jets. ASME Journal of Heat Transfer, vol. 117, pp. 878 (1995).

Patel, P. and Roy, S., Study of jet impingement heat transfer for varying fluid flow characteristics. ASME Fluids Engineering Division Summer Meeting, May 29 - June 1, New Orleans, Louisiana (2001).

Poh, H. J., Kumar, K. and Mujumdar A. S., Heat transfer from a pulsed laminar impinging jet. International Communication of Heat and Mass Transfer, vol. 32, Issue 10, pp. 1317-1324 (2005).

Polat, S., Huang, B., Mujumdar, A. S. and Douglas, W. J. M., Numerical flow and heat transfer under impinging jets: A review. Annual Review of $\mathrm{Nu}-$ merical Fluid Mechanics and Heat and Transfer, vol. 2, pp. 157-197 (1989).

Rahman, M. M., Lallave, J. C. and Kumar, A., Heat transfer from a spinning disk during semiconfined axial impingement from a rotating nozzle. 
International Journal of Heat and Mass Transfer, vol. 51, pp. 4400-4414 (2008).

Robinson, A. J. and Schnitzler, E., An experimental investigation of free and submerged miniature liquid jet array impingement heat transfer. Experimental Thermal and Fluid Science, vol. 32, pp. 1-13 (2007).

Roy, G., Nguyen, C. T. and Lajoie, P. R., Numerical investigation of laminar flow and heat transfer in a radial flow cooling system with the use of nanofluids. Superlattices and Microstructures, vol. 35, pp. 497-511 (2004).

Royne, A. and Dey, C. J., Effect of nozzle geometry on pressure drop and heat transfer in submerged jet arrays. International Journal of Heat and Mass Transfer, vol. 49, pp. 800-804 (2006).

Royne, A. and Dey, C. J., Design of a jet impingement cooling device for densely packed PV cells under high concentration. Solar Energy, vol. 81, pp. 1014-1024 (2007).

San, J. Y. and Lai, M., Optimum jet-to-jet spacing of heat transfer for staggered arrays of impinging air jets. International Journal of Heat and Mass Transfer, vol. 44, pp. 3997-4007 (2001).

Schutt, W., Continuous-flow jet-heating furnaces for heating and heat treatment of aluminium forgings and castings. Gaswaerme International, vol. 56, pp. 290-292 (2007).

Silverman, I. and Nagler, A., high heat flux cooling with water jet impingement. ASME Heat Transfer/ Fluids Engineering Summer Conference Charlotte, North Carolina, July. pp. 11-15 (2004).

Stevens, J. and Webb, B. W., Local heat transfer coefficients under an axisymmetric, single phase liquid jet. ASME Journal of Heat Transfer, vol. 113, pp. 71-72 (1991).

Sun, H., Ma, C. F. and Chen, Y. C., Prandtl number dependence of impingement heat transfer with circular free-surface liquid jets. International Journal of Heat and Mass Transfer, vol. 41, no. 10, pp. 1360-1363 (1998).

Sung, M. K. and Mudawar, I., Single-phase hybrid micro-channel/micro-jet impingement cooling. International Journal of Heat and Mass Transfer, vol. 51, pp. 4342-4352 (2008).

Teamah, M. A. and Farahat, S., Experimental and numerical heat transfer from impinging of single free liquid jet. Alexandria Engineering Journal, vol. 42, no. 5, pp. 559-575 (2003).

Vader, D. T., Incropera, F. P. and Viskanta, R., Local convective heat transfer from a heated surface to an impinging planar jet of water. International Journal of heat and Mass Transfer, vol. 34, no. 3, pp. 611-623 (1991).
Varella, D. A. and Leinhard, V. J. H., Development on non-linear waves on non-uniform axisymmetric film. Bulletin of American Physical Society, AI8, vol. 36, no. 10, $44^{\text {th }}$ Annual Meeting of Division on Fluid Dynamics, Scottsdale, November (1991).

Wang, Xiangqi, New approaches to micro-electronic component cooling. PhD Thesis in Mechanical Engineering, National University of Singapore, Singapore (2007).

Wang, Xiang-Qi and Mujumdar, A. S., A review on nanofluids - Part I: Theoretical and numerical investigations. Braz. J. Chem. Eng., vol. 25, no. 4, p. 613-630 (2008a).

Wang, Xiang-Qi and Mujumdar, A. S., A review on nanofluids - Part II: Experiments and applications. Braz. J. Chem. Eng., vol. 25, no. 4, p. 631-648 (2008b).

Wang, N. E., Zhang, L., Jiang, L., Koo, J. M., Maveety, J. G., Sanchez, E. A., Goodson, K. E. and Kenny, T. W., Micromachined jets for liquid impingement cooling of VLSI chips. Journal of Microelectromechanical Systems, vol. 13, No. 5, pp. 833-842 (2004).

Watson, E. J., The radial spread of a liquid jet over a horizontal plane. Journal of Fluid Mechanics, vol. 20, pp. 481-499 (1964).

Webb, B. W., Ma, C. F., Single-Phase Liquid Jet Impingement Heat Transfer. In: J. P. Hartnett (Ed.) Advances in Heat Transfer, vol. 26, pp. 105-217, Academic Press, NewYork (1995).

Wolf, D. H., Viskanta, R. and Incorporeal, F. P., Turbulence dissipation in a free surface jet of water and its effect on local impingement heat transfer from a heated surface, Part 2 - local heat transfer. Journal of Heat Transfer, vol. 117, pp. 95-103 (1995).

Womac, D. J., Ramadhyani, S. and Incropra, F. P., Correlating equations for impingement cooling of small heat sources with single circular liquid jets. ASME Journal of Heat Transfer, vol. 115, pp. 106-115 (1993).

Womac, D. J., Incropra, F. P. and Ramadhyani, S., Correlating equation for impingement cooling of small heat sources with multiple circular liquid jets. ASME Journal of Heat Transfer, vol. 116, pp. 482-486 (1996).

Woodfield, P. L., Monde, M. and Mozumder, M. K., Observations of high temperature impinging-jet boiling phenomena. International Journal of Heat and Mass Transfer, vol. 48, no. 10, pp. 2032-2041 (2005).

Yang, Y. T. and Lai, F. H., Numerical study of heat transfer enhancement with the use of nanofluids in radial flow cooling system. International Journal 
of Heat and Mass Transfer, vol. 53, pp. 58955904 (2010).

Yang, Y. T. and Lai, F. H., Numerical investigation of cooling performance with the use of $\mathrm{Al}_{2} \mathrm{O}_{3} /$ water nanofluids in a radial flow system. International Journal of Thermal Sciences, vol. 50, pp. 61-72 (2011).

Zhang, L. X. and Hu, G. X., Experimental study of heat transfer characteristics with impinging jet at curved surface. Journal of Chemical Industry and Engineering, vol. 56, pp. 1409-1412 (2005).
Zuckerman, N. and Lior, N., Jet impingement heat transfer: Physics, correlations, and numerical modeling. Advances in Heat Transfer, vol. 39, pp. 565-631 (2006).

Zuckerman, N. and Lior, N., Radial slot jet impingement flow and heat transfer on a cylindrical target. Journal of Thermophysics and Heat Transfer, vol. 21, No. 3, pp. 548-561 (2007).

Zeitoun, O. and Ali, M., Nanofluid impingement jet heat transfer. Nanoscale Research Letters, vol. 7, pp. 139 (2012). 